\title{
LA CERAMICA A VERNICE NERA DAL TOFET DI MOZIA: ATTESTAZIONI E IMPLICAZIONI SOCIO-ECONOMICHE NEL PERIODO POST-DIONIGIANO
}

\author{
Ilenia Melis* - Sapienza University of Rome
}

The aim of this study is examining the Black Glazed Pottery of the Tophet of Motya dating to the $4^{\text {th }}$ century BC. The analysis of the pottery repertoire is twofold: it allows to understand the meaning of the utilization of the Greek imports during the rituals, and it gives the opportunity for a reconsideration of the spread of Attic imports in Western markets and the role of Motya in these trades during the $4^{\text {th }}$ century $B C$.

Keywords: Black Glazed Pottery; Attic imports; ritual practices; Tophet; Motya

\section{INTRODUZIONE}

La ceramica a vernice nera rappresenta la classe di importazione maggiormente attestata a Mozia nel periodo successivo alla distruzione della città da parte di Dionigi di Siracusa nel 397/6 a.C. L'abbondante presenza di forme di imitazione siceliota rispetto al V secolo a.C. testimonia lo sviluppo di un fiorente mercato regionale sempre più in espansione che si sostituirà gradualmente nel periodo ellenistico alle botteghe attiche ${ }^{1}$. Accanto ad una gran messe di prodotti di imitazione coloniale si registra sul finire del V secolo a.C. e per tutto il secolo successivo una discreta presenza di importazioni attiche ${ }^{2}$. L'analisi del repertorio ceramico del Tofet contribuisce a chiarire quanto già delineato per i quartieri dell'abitato ${ }^{3} \mathrm{e}$ per il Santuario $\mathrm{C}^{4}$, gettando le premesse per una rinnovata riflessione sulla circolazione della ceramica attica nei mercati occidentali durante il IV sec. a.C. e sul ruolo commerciale rivestito da Mozia all'interno di queste nuove rotte.

\section{IL TOFET}

Il Tofet di Mozia è stato esplorato in una serie di campagne realizzate congiuntamente dalla Soprintendenza di Trapani e l'Università di Roma La Sapienza dal 1964 al 19735. Gli scavi condotti da Antonia Ciasca misero in luce circa i 2/3 del Tofet e chiarirono la planimetria delle strutture del santuario e la relazione stratigrafica con il campo d'urne (fig. 1) ${ }^{6}$. Gli scavi furono ripresi tra il 2009 e il 2014 dalla Missione Archeologica a Mozia,

* PhD Student in Archeologia Fenicio-Punica a "La Sapienza" Università di Roma. Un sentito ringraziamento al Prof. Lorenzo Nigro per avermi concesso lo studio di questi materiali e alla Prof.ssa Federica Spagnoli per avermi guidato nell'analisi del repertorio ceramico.

1 Il mercato regionale a cui si fa riferimento è quello della Sicilia occidentale. Tra i possibili centri produttori si segnalano: Himera, Termini Himerese, Monte Iato, Marineo ed Entella, Solunto, Selinunte e Lilibeo (Belvedere et al. 2006, 563-567).

2 Si tratta di un trend riscontrabile generalmente nei siti della Sicilia Occidentale (Bechtold 2007, 58).

Rocco 2007, 56-57.

Nigro 2009a, 708.

Nigro 2004a, 20-24.

$6 \quad$ Ciasca 1964; 1967; 1968; 1968-1969; 1969; 1970; 1971; 1972; 1972-1973; 1973; 1978; 1992; 2002; Ciasca et al. 1996; Nigro 2004 b.

ISSN 0393-0300; 2724-587X

e-ISSN 2532-5159

doi: 10.53131/VO2724-587X2021_6 
diretta da Lorenzo Nigro, al fine di investigare il limite meridionale del santuario e le strutture ad esso connesse (fig. 2) ${ }^{7}$. Il Tofet rimase in uso durante tutto l'arco temporale della città di Mozia e la stratigrafia elaborata dalla Ciasca scandisce i diversi momenti di vita del Santuario. Questa si compone di 7 macro-strati a cui corrispondono tre diverse fasi cronologiche (fig. 3). La Fase A (Strata VII-V, 750-550 a.C.) identifica il nucleo più antico del Santuario, occupato dal primo campo di urne; la Fase B (Strata IV-I,2, 550-397 a.C.) corrispondente al periodo di rinnovamento e restauro del santuario con relativo ampliamento del campo deposizionale verso est e l'erezione di nuove strutture sacre di servizio; la Fase C (Stratum I,1, post 397 a.C.) ultimo periodo occupazionale del Tofet di Mozia dopo la distruzione dionigiana del 397/6 a.C.

\section{LA CERAMICA A VERNICE NERA DALLO STRATO I,1 (MOZIA VIII, 400-300 A.C.)}

Vengono qui presi in esame $i$ frammenti ceramici rinvenuti sporadicamente all'interno degli strati deposizionali, non associati alle urne come elementi di corredo ma utilizzati durante specifiche pratiche rituali ${ }^{8}$. La ceramica a vernice nera rappresenta la classe di importazione ormai predominante nel IV secolo a.C. quando si assiste all'ellenizzazione del repertorio ceramico moziese ${ }^{9}$, testimoniata anche dal rinnovamento formale dei cinerari che comporta la sostituzione dell'olla biansata ${ }^{10}$ con forme di tradizione greca come la cytra o lo stamnos $^{11}$.

\subsection{La ceramica da mensa}

La ceramica da mensa rappresenta 1 ' $87 \%$ dei frammenti a vernice nera rinvenuti nel Tofet (fig. 4). La forma maggiormente attestata, sia di importazione che imitazione coloniale, è la coppetta incurving rim. Di 91 esemplari 36 sono di importazione attica (nn. 4-12) e 55 di imitazione coloniale (nn. 31-39). I frammenti sono riconducibili alla serie 2714 Morel $^{12}$, ispirati ai prototipi attici delle forme nn. 876, 870, 882 dell'Athenian Agora databili tra 400 e 350 a.C. ${ }^{13}$

La tipologia più antica documentata è quella delle coppe stemless (n. 28) di cui si conservano due frammenti di imitazione coloniale ispirati alla forma 515 dell'Athenian Agora e databili tra V e IV secolo a.C. ${ }^{14}$.

\footnotetext{
Nigro 2013; 2017, 70-73; 2018, 258; 2020. Il Tofet fu interessato contestualmente anche da opere di restauro (Nigro 2012, 207; Giglio 2012, 220-221).

8 Si riportano in tavola gli esemplari rappresentativi per ogni tipologia descritta di cui si dà il numero totale di attestazioni nel catalogo.

9 Orsingher 2013a, 693-695.

10 Sull'evoluzione formale delle urne del Tofet: Orsingher 2013b, 761; 2015, 565-567; 2016, 286-289.

11 Il dato conferma quanto già delineato per il V secolo a.C. nello studio della ceramica di importazione greca proveniente dal Tofet. Su un totale di 1814 frammenti schedati 1738 appartengono alla produzione a vernice nera mentre i restanti alla produzione figurata. All'interno di questo cospicuo gruppo dominano le forme potorie riconducibili alla pratica del banchetto $(77 \%)$ seguite dalle forme per versare $(16 \%)$ e dalle forme per usi speciali $(7 \%)$.

12 Morel 1981, 209, tav. 67:2714.

13 Sparkes - Talcott 1970, fig. 9:876, 870, 882

14 Sparkes - Talcott 1970, fig. 5:515.
} 
Alla prima metà del IV secolo a.C. sono ascrivibili tre coppe attiche tipo bolsal (n. 1), di cui si conserva solo il fondo, riconducibili al tipo 554 dell'Athenian Agora e datate al 375 a.C. ${ }^{15}$, tre coppe attiche del tipo broad rim (n. 2), riconducibili alla forma 783 dell'Athenian Agora ed un esemplare di imitazione siceliota (n. 29) ${ }^{16}$. Un unico esemplare attico di coppa shallow wall convex-concave profile (n. 14) è datato al 375 a.C. ${ }^{17}$.

Alla metà del IV sec. a.C. si ascrivono un frammento attico di coppa carenata (n. 15), del tipo 829 dell'Athenian Agora ${ }^{18}$, e un frammento di imitazione siceliota (n. 40) ispirato allo stesso tipo e riconducibile alla serie Morel $2744^{19}$. Un frammento (n. 41), databile al finire del IV secolo a.C. riconducibile alla serie Morel $2831^{20}$.

Tra i reperti più tardi, datati alla seconda metà del IV secolo a.C., vi sono cinque frammenti di coppa tipo outturned rim. Tre sono di importazione attica del tipo 802 dell'Athenian Agora (n. 3) mentre due di produzione siceliota sono riconducibili alla serie Morel 2672 (n. 30) ${ }^{21}$.

Due frammenti di coppa one handler, uno attico (n. 13) riconducibile al tipo 757 dell'Athenian Agora e uno siceliota (n. 43) della serie Morel 2625, completano il repertorio delle coppe ${ }^{22}$.

La seconda forma da mensa maggiormente attestata è quella degli skyphoi ${ }^{23} \mathrm{Si}$ tratta di 10 frammenti di skyphoi A di tipo attico, 7 dei quali riconducibili al tipo 349 dell'Athenian Agora ed inquadrabili al primo venticinquennio del IV secolo a.C. (nn. 16-17) e 3 frammenti più tardi (n. 18-19) riconducibili alla forma 352 e databili intorno al 330 a.C. A questi si aggiungono 11 frammenti di imitazione siceliota del tipo Morel 4373 e ispirati alla medesima forma (nn. 44-46) ${ }^{24}$.

I piatti sono rappresentai da un unico esemplare di imitazione siceliota (n. 47), della serie Morel $1128 \mathrm{a}^{25}$, ispirato al tipo attico thickned edge e datato al secondo venticinquennio del IV secolo a.C. ${ }^{26}$.

Completano l'analisi delle forme potorie un kantharos attico (n. 25) del tipo 617 dell'Athenian Agora e databile agli inizi del IV sec. a.C. ${ }^{27}$.

15 Sparkes - Talcott 1970, fig. 274, fig. 6:554.

16 Sparkes - Talcott 1970, 292, fig. 8:783. Il frammento siceliota è assimilabile morfologicamente alla serie Morel 2985 per quanto la datazione proposta dall'autore sia troppo tarda per l'esemplare moziese (Morel 1981, 245, tav. 85:2985c 1).

17 Sparkes - Talcott 1970, 295, fig. 8:822.

18 Sparkes - Talcott 1970, 295, fig. 8:829.

19 Morel 1981, 216, tav. 69:2744b 1. Per quanto la datazione proposta da Morel al II secolo a.C. sia troppo tarda per il frammento moziese.

20 Morel 1981, 230, tav. 7:2831a 2.

21 Sparkes - Talcott 1970, 293, fig. 8:802; Morel 1981, 204, tav. 65:2672b.

22 Sparkes - Talcott 1970, 290, fig. 8:757; Morel 1981, 195, tav. 61:2625c 1.

23 Lo skyphos è rappresentato nella quasi totalità delle attestazioni da fondi. Si propongono in tavola un orlo e un fondo attico del tipo 349, MT.71.85/33 e MT.68.112/8, e un orlo ed un fondo del tipo 352, MT.66.354 e MT.68.112/13 (Sparkes - Talcott 1970, 260, fig. 4:349, 352). Degli esemplari sicelioti si conserva solamente un frammento di orlo molto fratturato, MT.67.115/7, e dieci fondi esemplificati in tavola dal frammento MT.70.100/7.

24 Morel 1981, 311, tav. 131:4373a 2.

25 Morel 1981, 87, tav.4, serie 1128a.

26 Sparkes - Talcott 1970, 307, fig. 9:1016 


\subsection{La ceramica per usi speciali}

Le forme per usi speciali rappresentano il $13 \%$ del repertorio ceramico a vernice nera rinvenuto nel Tofet (fig. 4). La forma maggiormente attestata è quella delle lucerne di cui si conservano 6 esemplari. Quattro esemplari attici sono riconducibili alle varianti 23A e 23B della Howland e inquadrabili tra 410 e 475 a.C. (nn. 23-24) ${ }^{28}$. Due esemplari sicelioti (nn. 48-49), imitanti i tipi 23C e 23D della Howland, sono databili rispettivamente al secondo e al terzo venticinquennio del IV sec. a.C. ${ }^{29}$.

Alla produzione attica sono attribuibili tre frammenti di lekythoi aryballiche (nn. 2022). Un orlo riconducibile alla forma 1099 dell'Athenian Agora e databile ai decenni finali del V sec. a.C. - inizi IV sec. a.C. ${ }^{30}$. Un orlo del tipo 1123 dell'Athenian Agora rappresenta un tipo rinvenuto ad Atene in contesti di fine $\mathrm{V}$ sec. a.C. e ancora esportate in occidente tra 375 e 325 a.C. ${ }^{31}$. A questi si aggiunge una squat lekythos caratterizzata da vertical stribs e riconducibile al tipo 1129 dell'Athenian Agora ${ }^{32}$.

Due pareti strigilate di guttui del tipo askoide (nn. 26-27) ed un frammento di parete con gorgoneion realizzato a stampo databile al periodo ellenistico sono attribuibili alla produzione attica (fig. 5) ${ }^{33}$.

Tra le forme aperte di imitazione siceliota sono attestati un frammento di lopadion a vernice rossa (n. 50) ${ }^{34}$ e una lekanis (n. 51) della serie Morel 4713 ispirata alla forma 2222 dell'Athenian Agora inquadrabile nel primo venticinquennio del IV sec. a.C. ${ }^{35}$.

\section{DAL REPERTORIO CERAMICO ALLA RICOSTRUZIONE DEL RITUALE}

Il repertorio vascolare a vernice nera di IV secolo a.C. è composto per 1'87\% da forme per il banchetto. Come nei secoli precedenti, anche nel IV secolo a.C. si evidenzia un'abbondanza di forme potorie rispetto alle forme di grandi dimensioni utilizzate per la preparazione del banchetto ${ }^{36}$. Si registra una notevole incidenza di coppette incurving rim, databili alla prima metà del IV secolo a.C. e di skyphoi tipo A mentre attestazioni sporadiche di coppe outturned rim, coppe caranate, shallow wall, bolsal, broad rim, stemless e cup-skyphoi completano il repertorio delle forme potorie del Tofet. L'ampia varietà formale comprova quanto l'ideologia del banchetto funerario e del simposio dovessero costituire una parte fondamentale delle pratiche cultuali del Tofet tanto da generare una abbondante richiesta di vasellame da mensa. Trattandosi di un contesto sacro

Sparkes - Talcott 1970, 279, fig. 6:617.

Howland 1958, 57, tav. 7.36:216; 65, tav. 9.37:255.

Howland 1958, 60, tavv. 8, 36:229; 61-62, tavv. 8, 37:238.

Sparkes - Talcott 1970, 313, fig. 11:1099.

Sparkes - Talcott 1970, 315, fig. 11:1123. Da Cartagine (Chelbi 1992, 61, n. 477).

Sparkes - Talcott 1970, 315, tav. 28:1129.

Ciasca 1966, 40, tav. LII.

34 Questa forma, analoga al lopas attico, per le ridotte dimensioni ha la funzione di una forma per usi speciali equiparabile ad una pisside (Orsingher 2011, 126).

35 Morel 1981, 327, tav. 143:4713a; Sparkes - Talcott 1970, 322, tav. 40:1222.

36 Tanto nella produzione corinzia quanto in quella attica sono scarsamente attestate le grandi forme aperte utilizzate per la mescita del vino. Si registra infatti un solo frammento di cratere di produzione attico corinzia cinque frammenti a vernice nera e quattro a figure rosse. 
con carattere anche sepolcrale non dovevano mancare rituali connessi all'ideologia del banchetto funebre e pasti comunitari ripetuti ciclicamente così da mantenere sempre vivo il legame tra il defunto e la comunità. Le forme da mensa venivano però utilizzate anche durante le pratiche libatorie in onore della divinità e ad essa consacrate una volta ultimato il rituale. È il caso di quattro fondi di skyphos e di una coppa stemless rifunzionalizzati proprio a tal scopo (fig. 6). Si tratta infatti di cinque frammenti che perdono la loro destinazione d'uso originaria al fine di ricoprire nuovi scopi di natura pratica o cultuale ${ }^{37}$. Il fondo di coppa stemless $^{38}$ fu intenzionalmente forato così da essere utilizzato come versatoio per libare. La pratica libatoria si configura come un modo per celebrare l'aspetto ctonio del dio Baal e le forme da mensa riutilizzate come versatoi non sono altro che lo strumento a disposizione degli offerenti per entrare in contatto con la divinità. Rispetto alle forme rifunzionalizzate maggiori sono le attestazioni di frammenti defunzionalizzati che cessano il loro ciclo di vita senza essere reimpiegati, come nel caso dei fondi di skyphos ${ }^{39}$. Il vasellame defunzionalizzato rappresenta l'ultimo atto di una sorta di rituale di obliterazione che nel Tofet doveva rispecchiarsi con il rituale funerario. Lo stesso banchetto funebre può considerarsi l'atto conclusivo, di obliterazione, di tutta la prassi rituale che si svolgeva nel Tofet, dalla deposizione dell'infante alla periodica pratica simposiale in memoria del defunto. Dopo aver libato e consumato il vino si sceglie di rompere il vasellame usato durante la cerimonia e di dedicarlo alla divinità così da chiudere $\mathrm{e}$ suggellare il rituale funerario ${ }^{40}$. Se da un lato anche nel Tofet c'è un forte richiamo alla sfera ctonia e al dio Baal la pratica della defunzionalizzazione si erge anche come metafora della vita, del non ritorno che coinvolge tanto gli uomini quanto gli oggetti.

Le lucerne ed i guttui sono le forme per usi speciali maggiormente rappresentative della peculiarità dei rituali del Tofet. Entrambe ricoprono un significato fortemente evocativo: il richiamo al fuoco e all'arsione trasforma la lucerna da un mero oggetto utilizzato durante le pratiche cultuali ad un simbolo del rituale così da spiegarne la presenza continuata nel corso del tempo. Allo stesso modo il guttus rimanda alla sfera di significati legata alla figura dell'infante. La sua presenza in associazione a sepolture infantili sottolinea lo status dei defunti, quello di bambini morti in età perinatale ${ }^{41}$, senza escluderne un possibile utilizzo durante rituali di aspersione, rievocativi di quella funzione di versatoio goccia a goccia utilizzato nelle pratiche alimentari dei bambini.

\section{LE IMPORTAZIONI ATTICHE DAL TOFET}

L'analisi delle produzioni attestate nel Tofet suggerisce dei quesiti legati alla circolazione della ceramica a vernice nera nel periodo post-dionigiano: quanto di questi materiali è riconoscibile come di importazione attica e quanto invece appartiene alla

\footnotetext{
37 È un fenomeno comune sia nel mondo levantino che in quello greco e magnogreco. In proposito: Aubet 2006, 45-46; Parisi 2017, 502-503; Panvini 2010, 56; Elia 2003, 151-153. A Mozia è documentato anche nell'area sacra del Kothon (Spagnoli 2012, 40; Nigro 2004c, 55-57, 122-124; Mozia XI, 414, 442, 458, 474, 494; Nigro 2009a).

38 MT.68.30/14. il frammento si data alla fine del V secolo a.C. (Sparkes - Talcott 1970, 269, fig. 5:488)

39 MT.65.1112, MT.67.121/15, MT.68.12/11, MT.68.9/13, MT.73.19/7.

40 Parisi 2017, 555-556.

41 Vassallo 2016, 50.
} 
tradizione coloniale? Quali scelte e ideologie si celano dietro l'importazione di specifiche forme?

$\mathrm{Si}$ propone quindi in questa sede una preliminare distinzione tra la valenza delle importazioni attiche e quella delle fabbriche coloniali ${ }^{42}$. Durante il IV sec. a.C. le importazioni di ceramica a vernice nera subiscono un'inflessione rispetto al secolo precedente, a cui si accompagna un forte decremento di prodotti attici a favore di un mercato coloniale ormai sempre più fiorente (fig. 7$)^{43}$. Analizzando i prodotti attici si evidenzia una volontà ben precisa dietro l'importazione di specifiche forme legate forse al valore cultuale che questi vasi assumevano durante il rituale. Sono infatti le forme per usi speciali, per quanto numericamente inferiori rispetto alle forme da mensa, ad essere maggiormente rappresentative della produzione attica di IV secolo a.C. Le lekythoi aryballiche e le squat lekythoi inquadrabili nella prima metà del IV secolo a.C. sono tutte di importazione attica. Le lekythoi rappresentano un prodotto di pregio non solo per la loro funzione rituale ma anche per la scarsa diffusione nei mercati occidentali ${ }^{44}$. Allo stesso modo i pochi frammenti di parete di guttus del tipo askoide sono riconducibili tutti alla produzione attica. Nonostante non si possa risalire al tipo preciso, essi sono un prodotto esportato nei mercati occidentali e molto apprezzato nei siti punici di Sicilia e Sardegna ${ }^{45}$.

Rappresentativi della produzione attica di IV secolo a.C. sono anche i pochi frammenti di lucerna rinvenuti nel Tofet. Rispetto al secolo precedente si assiste ad una drastica riduzione di questa forma, flessione riscontrata anche in altri contesti moziesi ${ }^{46} \mathrm{e}$ in Sardegna $^{47}$. Si tratta infatti di una forma non particolarmente esportata nei mercati

42 Tale distinzione viene elaborata su uno studio preliminare del materiale ceramico, basato sull'analisi degli impasti e sulla qualità dei rivestimenti in attesa di future analisi di tipo archeometrico. Vengono quindi definite come coloniali le produzioni riconosciute come chiaramente non attiche ed attribuibili a botteghe siceliote o dell'Italia meridionale.

43 Data la continuità d'uso del santuario il fenomeno è facilmente visibile nel Tofet dove tra V e IV secolo a.C. si assiste ad un'inflessione delle importazioni di ceramica a vernice nera di circa il $30 \%$, ma una situazione analoga è attesta anche per altre aree dell'isola.

44 In Spagna come in Sardegna costituiscono circa il 2\% delle importazioni attiche (Tronchetti 2020, 279). I frammenti attici del Tofet sono confrontabili con i pochi esemplari di globular lekythoi del Sec e con le squat lekythoi (Cerda 1987a, 372, 375:376; 1987b, 56-57) e con l'esemplare Cartaginese (Chelbi 1992, 61, n. 477).

45 In Sicilia sono numerose le attestazioni di produzione coloniale: da Palermo (Di Stefano 1998, 159, 186, $\mathrm{n}$ 211; 2009, 122, n. 54; 160, n. 9; 161, n. 25; 164, n. 36) e da Lilibeo (Di Stefano 1993, fig. 38:2, tav. XLV:1) In Sardegna l'askos-guttus è maggiormente apprezzato come contenitore di olii della lekythos aryballica (Tronchetti 2020, 279).

46 Nelle ultime fasi di frequentazione della Casa del Sacello domestico della Zona D sono stati rinvenuti due frammenti di tradizione siceliota (Rocco 2007, 56) ed un unico esemplare riconoscibile come attico (Rocco 2004, 223, MD.02.265/24); dalla Casa dei Mosaici provengono tre frammenti di tradizione siceliota del Tipo 23 della Howland (Acquaro - Del Vais - Secci 2007, 139-140, fig. 15:107-109); dall'Abitato A tre frammenti databili tra la fine del V-inizi IV sec. a.C. e sei frammenti di pieno IV sec. a.C. (Toti 2002a, 310) mentre dalla Fortezza Occidentale (Rocco 2011, 111)

47 Una situazione analoga si riscontra anche in Sardegna, specialmente nella Necropoli e nel Tofet di Tharros, dove viene interpretata come un fenomeno legato ad un possibile cambiamento culturale, riscontrabile soprattutto per quanto concerne le pratiche rituali. Al contempo tale cambiamento culturale è interpretabile anche alla luce di una omologazione del repertorio formale connessa alla politica cartaginese durante la seconda metà del IV secolo a.C. (Madau 1989, 85; 2000, 103.) 
occidentali e scarsamente attestata nei siti punici ${ }^{48}$, la cui richiesta viene sopperita da forme di imitazione coloniale oppure dalla stessa produzione punica. Nel Tofet dove forte è la simbologia della luce e dell'arsione, vengono quindi riservati i pochi esemplari attici che circolano a Mozia, proprio in virtù del pregio conferito a queste forme per via della loro scarsa diffusione nei mercati occidentali.

Le bolsal, le coppe shallow wall e le outturned rim rappresentano le forme potorie che pur meno significative quantitativamente sono quasi unicamente attestate da esemplari attici. Le coppe bolsal rappresentano una delle forme attiche maggiormente importate in ambiente punico durante il IV secolo a.C. ${ }^{49}$ mentre le coppe shallow wall sono raramente attestate nei siti punici della Sicilia e nel Mediterraneo centrale ${ }^{50}$. Si tratta infatti di una forma che raggiunge le due aree periferiche dell'Attica, del Danubio e del Mar Nero ad est e i mercati iberici ad ovest dove sono attestate anche delle produzioni di imitazione locale caratterizzate da argilla di colore grigio ${ }^{51}$. Allo stesso modo le coppe outturned rim, per quanto rappresentino una forma standardizzata sul finire del IV secolo a.C., non sono molto attestate a Mozia. Si tratta invece di una forma documentata in ambiente lilibetano e palermitano $^{52}$, nei siti della Sardegna e a Cartagine ${ }^{53}$, destinata a raggiungere i mercati iberici $^{54}$. Si tratterebbe di due forme che per la loro origine e per la scarsa circolazione in ambiente punico sono da considerare come prodotti di pregio il cui uso viene riservato quindi all'ambito cultuale. Stesso valore di prodotto di pregio potrebbe essere ricoperto dallo skyphos. Durante il IV secolo a.C. gli skyphoi sono scarsamente attestati nell'abitato moziese $^{55}$ pur trattandosi di una forma ampiamente diffusa e imitata nei siti punici d'occidente $^{56}$. La limitata presenza di skyphoi porterebbe a ritenere questa forma come

48 Gli esemplari moziesi trovano confronto con i frammenti rinvenuti nella necropoli punica di Palermo (Di Stefano 2009, tav. X:1-3), nel relitto del Sec (Cerda 1987a, 377-383) e nella Necropoli di Tuvixeddu, dove sono documentate solo 12 lucerne databili al IV secolo a.C. (Tronchetti 2016, 87).

49 Dalla Sardegna (Madau 2000, 100, fig.1, tipo 2a); dalle Baleari (Cerda 1987a, 231, fig. 8:b); da Cartagine dove rappresenta la forma più attesta nei contesti funerari di IV secolo a.C. (Chelbi 1992, 56, n. 347)

50 Il tipo maggiormente attestato è una rielaborazione senza risalto prodotta in ambiente coloniale. Nella Necropoli di Lilibeo è attestato solamente il tipo dalla carena marcata e senza risalto (Di Stefano 1992, tav. XXIX, fig. 2:7-11; 1993, fig. 29:7-11; Bechtold 1999, 58, tav. I:1-2.). Tipi analoghi provengono dalla Necropoli di Palermo?(Di Stefano 2009, 221, tav. XIII:4-5). Da Cartagine esemplari carenati di imitazione locale (Chelbi 1992, 36, n. 72) e con risalto (Chelbi 1992, 39, n. 177).

51 Cerda 1987a, 355-357, fig. 74.

52 Da Lilibeo (Di Stefano 1992, tav. XXVIII, fig. 2:9-13; 1993, fig. 30:9-13; Bechtold 1999, 63, tav. III:35-37). Dalla Necropoli di Palermo (Gli esemplari coloniali: Di Stefano 1998, 288, VG. 99; 2009, 80, n. 5; 160, n. 19; 165, nn. 42-43; 168, nn. 58-59).

53 Per Nora si veda: Bartoloni - Tronchetti 1981, 123, fig. 7:35.6.1; per Olbia: Corrias 2012, 289, fig. 4b; per la Necropoli di Tuvixeddu: Tronchetti 2016, 98, figg. 3-5. A Cartagine furono rinvenuti sia frammenti attici che di imitazione locale (Chelbi 1992, nn. 12-13, 96-102).

54 Cerda 1987a, 259-263.

55 Pochi frammenti provengono dalla Casa dei Mosaici (Acquaro - Del Vais - Secci 2007, 132, fig. 7:43-45, fig. 9:54-55), dalla Zona E (Famà - Toti 2000, 458), dall'Abitato A (Michelini 2002b, 188, tav. 8:89-91, tav. 9:9799), dai livelli di distruzione della Casa del Sacello domestico (Rocco 2004, 222; 2007, 56, nota 148).

56 Dalla Necropoli di Lilibeo (Di Stefano 1992, tav. XXX:1, 4-5; 1993, fig. 36:5-6; Bechtold 1999, tav. V:48); da Palermo (Di Stefano 1996, 692, fig. 3:2; 1998, 284, VG. 132; per gli esemplari coloniali: Di Stefano 2009, 220, tav. XII:6; 55, n. $1 ; 112$, n. 6; 116, n. 25; 142, n. $18 ; 155$, n. $2 ; 161$, n. 22; 168, n. 56; per gli esemplari attici: Di Stefano 2009, 118, n. 33). Da Solunto (Tardo 1997, 91, fig. 15:37; 2005, 683, fig. 3). 
riservata all'ambito cultuale mentre in ambiente domestico la medesima funzione doveva essere assolta da forme puniche di uso quotidiano. L'attestazionenel Tofet di pochi frammenti databili al finire del IV secolo a.C. sarebbe una ulteriore conferma di quanto ipotizzato. Le coppette incurving rim sono la forma maggiormente attestata, nella quasi totalità di produzione coloniale e solo in minima parte di importazione attica ${ }^{57}$. Si tratta infatti di una forma ampiamente imitata in ambiente siceliota e magno-greco e diffusa nei siti punici della Sicilia, dove a fianco di pochi esemplari attici si registra un gran numero di forme di imitazione ${ }^{58}$.

\section{CONCLUSIONI}

L'analisi della ceramica a vernice nera e delle forme di importazione attica rinvenute nel Tofet durante il IV secolo a.C. permette di riflettere ancora una volta sul ruolo svolto dalla Sicilia punica, ed in particolare da Mozia, nelle rotte commerciali che, partendo da Atene, attraversavano il Mediterraneo alla volta dei mercati occidentali. Analizzando il repertorio vascolare del Tofet si registra infatti, a fianco di una cospicua e predominante presenza di ceramiche prodotte nei mercati coloniali, una presenza non trascurabile di prodotti di importazione attica. Questo fenomeno si pone da un lato in controtendenza con quanto evidenziato nei siti del Mediterraneo centrale, dove durante il IV secolo a.C. si assiste ad un crollo delle importazioni attiche a favore di uno sviluppo di botteghe locali ${ }^{59}$, dall'altro sembra chiaramente leggibile alla luce del ruolo da sempre svolto da Mozia come testa di ponte tra l'Africa e le Spagna ${ }^{60}$. La rotta africana ripristinata da Atene durante il IV secolo a.C. porta ad una riapertura dei mercati iberici il cui raggiungimento non poteva non coinvolgere i siti punici della Sicilia e della Sardegna ${ }^{61}$. Lekythoi aryballiche, guttui del tipo askoide, bolsal, coppe outturned rim e shallow wall rappresentano i prodotti attici maggiormente documentati nei centri iberici e a Cartagine stessa. L'attestazione di tali forme anche a Mozia porta quindi a supporre che l'isola dovesse essere almeno toccata da

57 L'abbondanza di coppette incurving rim comprova l'ampia diffusione di questa forma a Mozia dove sono maggiormente attestati i prodotti sicelioti. Dalla Zona D (Rocco, 2004, n. 222), dalla Zona E (Famà - Toti 2000, 458-459, tav. XCI:3), dall'Abitato A (Michelini 2002b, 178-182). Dalla Fortezza Occidentale la gran messe di coppette con orlo rientrante rinvenuta negli strati di crollo della corte e degli altri ambienti è interpretata come un accumulo di materiali immagazzinati e deposti come offerte piuttosto che utilizzati come vasellame da mensa (Rocco 2011, 110-111).

58 Questa forma è ampiamente diffusa nella necropoli di Lilibeo con una duplice variante. La prima caratterizzata da orlo rientrante e ampio piede a sezione circolare, il secondo da stretto piede ad anello. Gli esemplari moziesi trovano stretta corrispondenza con quest'ultimo tipo (Di Stefano 1992, tav. XXVIII, fig. 2:5-8; tav. XXIX, fig. 1:5, 11; fig. 2:1-2; 1993, fig. 28:5, 10, 12; fig. 29:1-2; Bechtold 1999, tav. I:5, 7, 13.). Nella Necropoli di Solunto gli esemplari di imitazione coloniale costituiscono 1'85,5 5 delle coppette incurving rim rinvenute nei corredi delle sepolture databili alla seconda metà del IV secolo a.C. mentre scarsi frammenti sono databili alla prima metà del IV secolo a.C. (Tardo 1997, 88, fig. 12:46-47, 49; 2005, 681, fig. 2). Dalla Necropoli di Palermo (Di Stefano 1996, fig. 5:4; 1998, 288, VG 36, VG. 153; 2009, 221, tav. XIII:23,$6 ; 61$, n. $22 ; 66$, n. $51 ; 80$, n. $13 ; 114$, n. $12 ; 117$, n. $31 ; 124$, n. $67 ; 155$, n. $1 ; 161$, nn. 23,$26 ; 163$, n. 27).

59 Giudice 2001, fig. 1; 2006.

60 Si registra infatti un netto contrasto tra l'area punicizzante e il resto del Mediterraneo occidentale dove la ceramica attica a vernice nera risulta scarsamente diffusa (Morel 2000, 9).

61 Giudice - Giudice 2007, 321-322 con bibliografia precedente; Giudice 2012, 30. 
questa rotta, se non direttamente coinvolta nello smistamento di prodotti attici in questa area del Mediterraneo.

La significativa presenza di queste importazioni fornisce un'ulteriore conferma di come Mozia non subisca un crollo repentino dopo la distruzione del 397/6 a.C. ad opera di Dionigi di Siracusa ma si configuri come una realtà dai caratteri rinnovati ${ }^{62}$. Alcuni settori di abitato furono rioccupati a scopi produttivi ${ }^{63}$, la Fortezza Occidentale fu ricostruita ${ }^{64}$, il Tofet e il Cappiddazzu ${ }^{65}$ rimasero in uso mentre nell'area sacra del Kothon un nuovo santuario a cielo aperto sorse sulle rovine del precedente tempio di Baal ${ }^{66}$. Il repertorio ceramico del Santuario $\mathrm{C} 3{ }^{67}$ presenta numerose analogie con quello del Tofet, segno di una committenza che doveva essere comune. Lekythoi, coppe e skyphoi, guttui rappresentano le forme maggiormente attestate non solo nei due santuari moziesi ma anche nei corredi della Necropoli di Lilibeo. La continuità culturale tra le due città è spiegabile proprio alla luce degli eventi che coinvolsero Mozia nel 397/6 a.C. Dopo la distruzione della città i moziesi superstiti si rifugiarono sulla costa marsalese, ri-fondando Lilibeo che in età ellenistica diverrà una fiorente città dell'epicrazia cartaginese in Sicilia occidentale ${ }^{68}$. Nei decenni successivi alla distruzione i nuovi abitanti lilibetani mantennero un forte legame con Mozia che si tradusse nella continuità di frequentazione per circa un secolo dei principali santuari dell'isola; tra questi, il Santuario C3 e il Tofet divennero un luogo dove commemorare la caduta della città per mano dei siracusani.

Le importazioni attiche rinvenute nel Tofet si configurano quindi come particolari prodotti di pregio non solo per la valenza ricoperta durante le pratiche rituali ma anche per la ridotta quantità di ceramica attica che circola nei mercati occidentali. La ceramica attica attestata più raramente nei contesti d'abitato, dove per uso quotidiano si prediligono forme puniche oppure provenienti dalle botteghe siceliote, si trasforma nel Tofet, ed in generale nei santuari moziesi, in un prodotto di pregio che testimonia la rinnovata capacità di Mozia di inserirsi nel nuovo scenario commerciale mediterraneo, gettando le premesse per una rinnovata riflessione sul ruolo svolto da Mozia e più in generale della Sicilia punica nell'esportazione di prodotti attici nei mercati occidentali durante il IV secolo a.C.

62 Nigro 2015, 16.

63 Nella Zona D e B sono attestati forni per la fusione dei metalli (Nigro 2007, 17-18). L'area industriale, situata nel quadrante settentrionale dell'isola, rimase ancora attiva con gli impianti per la tintura con la porpora (Tusa 1973, 38-56; 1978, 65-90; Spanò Giammellaro 2000) mentre fornaci per ceramica furono realizzate lungo le mura (Toti 2002b).

64 Nigro 2009b, 553-554; Nigro - Melandri 2011, 76-102.

65 Il Tempio del Cappiddazzu venne ulteriormente monumentalizzato ampliando la facciata anteriore attraverso l'aggiunta di un portico che fu successivamente inglobato nelle strutture romane (Nigro - Spagnoli 2004, 5860; Nigro 2009c, 247-251). Per le trasformazioni successive (Nigro 2015, 19-21).

66 Nigro 2004c, 53-67; 2005, 60-92.

67 Nigro 2009a, 706-708.

68 Diodoro, XXII, 10,4; Bisi 1968, 29-31; Di Stefano 1984, 14-15; Garbini 1993, 67-72. 


\section{BIBLIOGRAFIA}

Aubet, M.E.

2006 Burial, symbols and mortuary practices in a Phoenician tomb: E. HERRING - I. LEMOS - F. Lo Schiavo - L. VagnetTi - D. Ridgeway (eds.), Across Frontiers. Etruscans, Greeks, Phoenicians and Cypriots. Studies in honor of David Ridgeway and Francesca Romana Serra Ridgeway (Accordia Specialist Studies on the Mediterranean 6), London 2006, pp. 37-47.

ACQuaro, E. - Del Vais, C. - SecCi, R.

2007 Mozia: "la casa dei mosaici". Scavi 1985. Edizione dei materiali ceramici: Byrsa. Rivista semestrale di arte, cultura e archeologia del Mediterraneo punico 1-4/2004-2005 (2007), pp. 125-228.

Bartoloni, P. - Tronchetti, C.

1981 La Necropoli di Nora (Collezione di Studi Fenici 12), Roma 1981.

BECHTOLD, B.

1999 La ceramica a vernice nera: B. BEChtold (ed.), La Necropoli di Lilybaeum (Regione siciliana. Assessorato dei Beni Culturali, Ambientali e della Pubblica Istruzione), Roma 1999, pp. 58-114.

2007 Alcune osservazioni sui rapporti commerciali fra Cartagine, la Sicilia Occidentale e la Campania (IV-metà del II sec. a.C.): nuovi dati basati sulla distribuzione di ceramiche campane e nordafricane/cartaginesi: Bulletin Antieke Beschaving 82 (2007), pp. 51-76.

Belvedere, O. - Burgio, A. - Iliopoulos, I. - Montana, G. - Spatafora, F.

2006 Ceramica a vernice nera di età ellenistica da siti della Sicilia nord-occidentale: considerazioni tipologiche e analisi archeometriche: Mélanges de l'École française de BISI, A.M. Rome. Moyen Âge (Antiquité 118, 2), Roma 2006, pp. 549-571.

1968 Il ruolo di Lilibeo nel quadro della cultura artistica della Sicilia Punica: Sicilia Archeologica 2 (1968), pp. 29-45.

CERDA, D.

1987a La cerámica ática de barniz negro: A. ArRIBAS - Mª.G. TRIAS - D. CERDA - J. De LA ROZ (eds.), El barco de el Sec (Calvià, Mallorca). Estudio de los materiales, Mallorca 1987, pp. 197-385.

1987b El Sec: La cerámica ática de barniz negro y las ámphoras: P. Rouillard - M.C. Villanueava-Puig (éd.), Grecs et Ibères au IVe siècle avant Jésus-Christ: sous la direction de Pierre Rouillard et Marie-Christine Villanueva-Puig (Revue des Études Anciennes 89, n. 3-4.), Bordeaux 1987, pp. 51-92.

CHELBI, F.

1992 Céramique à vernis noir de Carthage (Institut national d'archéologie et d'art), Tunis 1992.

CIASCA, A.

1964 Il Tophet. Lo scavo del 1964: A. CiasCA - M. Forte - G. Garbini - S. MosCati - B. PUGLIESE - V. TuSA (edd.): Mozia-I. Rapporto preliminare della Missione archeologica della Soprintendenza alle antichità della Sicilia occidentale e dell'Università di Roma (Studi semitici 12), Roma 1964, pp. 47-60.

1966 Il Tophet. Lo scavo del 1965: A. CiASCA - M. ForTe - G. GARbini - V. TuSA - A. TusA CUTRONI - A. VERGER (edd.), Mozia-II. Rapporto preliminare della Missione archeologica della Soprintendenza alle Antichità della Sicilia Occidentale e dell'Università di Roma (Studi Semitici 19), Roma 1966, pp. 27-53. 
1967 Il Tophet. Lo scavo del 1966: I. Brancoli - A. CiasCA - G. Garbini - B. Pugliese - V. TUSA - A. TUSA CUTRONI - A. Verger (edd.), Mozia-III. Rapporto preliminare della Missione archeologica della Soprintendenza alle Antichità della Sicilia occidentale e dell'Università di Roma (Studi Semitici 24), Roma 1967, pp. 11-25.

1968 Il Tophet. Lo scavo del 1967: A. CiasCA - G. GARbini - P. Mingazzini - B. Pugliese - V. TUSA (edd.), Mozia-IV. Rapporto preliminare della Missione archeologica della Soprintendenza alle Antichità della Sicilia occidentale e dell'Università di Roma (Studi Semitici 29), Roma 1968, pp. 25-53.

1968-1969 Scavi recenti al Tophet di Mozia: Kokalos 14-15 (1968-1969), pp. 315-320.

1969 Il Tophet. Lo scavo del 1968: A. CiasCa - M.G. Guzzo Amadasi - G. MatthiaE Scandone - B. Olivieri Pugliese - A. Tusa Cutroni - V. Tusa (edd.), Mozia-V Rapporto preliminare della Missione congiunta con la Soprintendenza alle Antichità della Sicilia occidentale (Studi Semitici 31), Roma 1969, pp. 35-52.

1970 Il Tofet. Lo Scavo del 1969: A. CiASCA - M.G. GuZZo - S. Moscati - V. TuSA (edd.), Mozia-VI. Rapporto preliminare della Missione congiunta con la Soprintendenza alle Antichità della Sicilia occidentale (Studi Semitici 37), Roma 1970, pp. 63-81.

1971 Sul Tofet di Mozia: Sicilia Archeologica 14 (1971), pp. 11-15.

1972 Il Tophet. Lo scavo del 1970: A. CiasCA - G. MatTHiae SCAdONE - S. Moscati - V. Tusa - A. TUSA CUTRONI (edd.), Mozia-VII. Rapporto preliminare della Missione congiunta con la Soprintendenza alle Antichità della Sicilia occidentale (Studi Semitici 40), Roma 1972, pp. 87-100.

1972-1973 Sul Tofet di Mozia: Kokalos 18-19 (1972-1973), pp. 411-414.

1973 Il Tophet. Lo scavo del 1971: A. CiASCA - V. TUSA - M.L. UberTi (edd.), Mozia-VII. Rapporto preliminare della Missione congiunta con la Soprintendenza alle Antichità della Sicilia occidentale (Studi Semitici 45), Roma 1973, pp. 57-71.

1978 Il Tophet. Lo scavo del 1972 e 1973: A. CiASCA - G. COACCI POLSELLI - N. CuOMo DI Caprio - M.G. Guzzo Amadasi - G. Matthiae Scandone - V. Tusa - V. Cutroni Tusa M.L. Uberti (edd.), Mozia-IX. Rapporto preliminare della Missione congiunta con la Soprintendenza alle Antichità della Sicilia occidentale (Studi Semitici 50), Roma 1978, pp. 125-143.

1992 Mozia: sguardo d'insieme sul tofet: Vicino Oriente VIII (1992), pp. 113-155.

2002 Archeologia del Tofet: A. GONZALES - L. RUIZ CABRERA (hrsgg.), Molk als opferbegriff im unischen und hebraischen, und das Ende des gottes Moloch. Molch como concepto de sacrificio punico y hebreo y el final del dios Moloch, Madrid 2002, pp. 121-140.

Ciasca, A. - Di Salvo, R. - Castellino, M. - Di Patti, C.

1996 Saggio preliminare sugli incinerati del Tofet di Mozia: Vicino Oriente 10 (1996), pp. $317-$ 348 .

CORRIAS, F.

2012 La ceramica a vernice nera da Olbia, Sardegna. Tra produzioni attiche e italiche, alcune forme di transizione: G. GuARDUCCI - S. VALENTINI (edd.), Il futuro dell'archeologia. Il Contributo dei Giovani Ricercatori (Tuscania (VT), 12-15 maggio 2011). Atti del IV convegno nazionale dei giovani archeologici, Roma 2012, pp. 284-293.

Di SteFano, C.A.

1984 Cenni storici: C.A. Di STEFANO (a cura di), Lilibeo. Testimonianze archeologiche dal IV sec. a.C. al V sec. d.C. Marsala Chiesa del Collegio dal 3 dicembre 1984, Marsala 1984, pp. 14-17.

1992 Ceramica a vernice nera del IV e III sec. a.C. da Lilibeo: una breve nota: L. BIONDI - C. Cassanelli (edd.), Giornate Internazionali di Studi sull'Area Elima (Gibellina, 19-22 settembre 1991), Pisa - Gibellina 1992, pp. 257-263. 
1993 Lilibeo Punica (Itinerari fenici e punici 12), Marsala 1993.

1996 Ceramiche a vernice nera dalla necropoli punica di Palermo: E. ACQUARO (ed.), Alle soglie della classicità. Il Mediterraneo tra tradizione e innovazione. Studi in onore di S. Moscati, Pisa - Roma 1996, pp. 679-694.

1998 La ceramica a vernice nera: C.A. Di StEFANO - P. TODARO - P. ANELlo - R. De SimONE O. Belvedere - I. Tamburello - R. Camerata Scovazzo - G. Castellana - R. Di Salvo - G. SarÀ - N. Allegro - A. Villa - A. Merra - G. Falsone - S. Ruvituso - C. Biagini - L- Gandolfo (edd.), Palermo Punica. Catalogo della Mostra. Museo Archeologico Regionale "Antonio Salinas" 6 dicembre 1995-30 settembre 1996, Palermo 1998, pp. 280-293.

2009 La Necropoli Punica di Palermo. Dieci anni di scavi nell'area della caserma Tukory (Sicilia Antiqua 4), Pisa - Roma 2009.

ELIA, D.

2003 L'offerta di sostanze alimentari liquide presso la tomba e l'uso rituale del cratere: Orizzonti 4 (2003), pp. 145-154.

FAMÀ, M.L. - TOTI, P.

2000 I materiali dalla Zona E dell'Abitato di Mozia. Prime considerazioni: A. CorReTTI (ed.), Terze Giornate Internazionali di Studi sull'Area Elima (Gibellina-Erice-Contessa GARBINI, G. Entellina, 23-26 ottobre 1997). Atti I, Pisa - Gibellina 2000, pp. 451-478.

1993 La caduta di Mozia: V. Tusa (ed.), Studi sulla Sicilia Occidentale in Onore di Vincenzo Tusa, Padova 1993, 67-72

GIGLIO, R.

2012 Il restauro e la valorizzazione del Tofet di Mozia: C. Ampolo (ed.), Sicilia Occidentale. Studi, rassegne e ricerche, Atti delle Settime Giornate Internazionali di Studi sull'area Elima e la Sicilia Occidentale nel contesto mediterraneo (Erice 2009) (Seminari e GIUDICE, G. Convegni 29), Pisa 2012, pp. 219-223.

2001 La ceramica attica del IV sec. a.C. in Sicilia ed il problema della formazione delle officine locali: N. Bonacasa - L. BracCesi - E. De Miro (edd.), La Sicilia dei due Dionisi, Atti della settimana di studio, Agrigento 24-28 febbraio 1999 (Progetto Akragas 2), Roma 2002, pp. 169-201.

2006 La ceramica attica del IV secolo e i clienti delle due Sicilie: J. DE LA GenIÈRE (éd.), Les clients de la céramique grecque. Actes du Colloque de l'Académie des Inscriptions et Belles-Lettres, Paris, 30-31 janvier 2004 (Cahiers du Corpus Vasorum Antiquorum 1), Paris 2006, pp. 93-95.

2012 Vedere il vaso attico: costruzione del quadro di riferimento delle forme dal 635 al 300 a.C.: S. SCHMIDT - A. STÄHLI (hrsgg.), Vasenbilder im kulturtransfer. Zirkulation und Rezeption griechischer Keramik im Mittelmeerraum (Beihefte zumVasorum Antiquorum 5), Munich 2012, pp. 27-34.

GiUdice, F. - GiUdice, G.

2007 Le grandi rotte della ceramica attica: riflessioni sui punti di snodo: G. SENA CHIESA (ed.), La Collezione di vasi Intesa Sanpaolo e i nuovi indirizzi di ricerca, Milano 2007, pp. 311334.

HowLAND, R.H.

$1958 \quad$ Greek Lamps and Their Survivals (Athenian Agora IV), Princeton 1958.

MADAU, M.

1989 Importazioni attiche da Tharros: Studi di Egittologia ed Antichità Puniche 4 (1989), pp. 73-87. 
2000 La ceramica attica di Tharros: le nuove stratigrafie dalla città fenicia del Sinis: B. SABATtini (éd.), La céramique attique du IVe siècle en Méditerranée occidentale. Actes du colloque international (Arles, 7-9 décembre 1995) (Collection du Centre Jean Bérard MiCHELINI, C. 19), Napoli 2000, pp. 99-104.

2002 Ceramica a vernice nera: M.L. FAmÀ (ed.), Mozia. Gli scavi nella zona A dell'abitato (Collana di archeologia del centro internazionale di Studi Fenici, Punici, Romani, Comune di Marsala 1). Bari 2002, pp. 165-202.

MOREL, J.P.

1981 La céramique campanienne : les formes (Bibliothèque des Écoles françaises d'Athènes et de Rome 244), Roma 1981.

2000 La céramique attique à vernis noir du IV ${ }^{\mathrm{e}}$ siecle: position des problèmes: B. SABBATtiNI (éd.), La céramique attique du IVe siecle en Méditerranée occidentale. Actes du colloque international organisé par le Centre Camille Jullian (Arles, 7-9 décembre 1995) (Publications du Centre Jean Bérard 19), Napoli 2000, pp. 1-31.

NigRo, L.

2004a Gli scavi di Antonia Ciasca al Tofet e alle mura (1964-1993): L. NigRo - G. RossonI (edd.), «La Sapienza» a Mozia. Quarant'anni di ricerca archeologica, 1964-2004. Catalogo della mostra, Università di Roma «La Sapienza», Facoltà di Scienze Umanistiche, Museo dell'Arte Classica, 27 febbraio - 18 maggio 2004 (Quaderni di Archeologia Fenicio-Punica, Colour Monograph 1), Roma 2004, pp. 20-29.

2004b Il Tofet: L. Nigro - G. Rossoni (edd.), «La Sapienza» a Mozia. Quarant'anni di ricerca archeologica, 1964-2004. Catalogo della mostra, Università di Roma «La Sapienza», Facoltà di Scienze Umanistiche, Museo dell'Arte Classica, 27 febbraio - 18 maggio 2004 (Quaderni di Archeologia Fenicio-Punica, Colour Monograph 1), Roma 2004, pp. 34-45.

2004c Zona C. Il Kothon. Il Santuario C3: architettura e ritrovamenti: L. NigRo (ed.), Mozia-X. Zona C. Il Kothon. Zona D. Pendici occidentali dell'Acropoli. Zona F. La porta Ovest. Rapporto preliminare della XII campagna di scavi (2002) condotte congiuntamente con il Servizio Beni Archeologici della Soprintendenza Regionale per i Beni Culturali e Ambientali di Trapani (Quaderni di Archeologia Fenicio-Punica I), Roma 2004, pp. 5367.

2005 Il santuario C3: architettura e ritrovamenti: L. NigRo (ed.), Mozia-XI. Il Tempio del Kothon. Rapporto preliminare delle campagne di scavi XXIII e XXIV (2003-2004) condotte congiuntamente con il Servizio Beni Archeologici della Soprintendenza Regionale per i Beni Culturali e Ambientali di Trapani (Quaderni di Archeologia FenicioPunica II), Roma 2005, pp. 60-92.

2007 La Stratigrafia. Zona D. Le Pendici Occidentali dell'Acropoli: La "Casa del Sacello Domestico" e il "Basamento Meridionale": L. Nigro (ed.), Mozia-XII. Zona D. Le Pendici Occidentali dell'Acropoli: La "Casa del Sacello Domestico", il "Basamento Meridionale" e il Sondaggio I. Rapporto preliminare delle campagne di scavi XXIII e XXIV (2003-2004) condotte congiuntamente con il Servizio Beni Archeologici della Soprintendenza Regionale per i Beni Culturali e Ambientali di Trapani (Quaderni di Archeologia Fenicio-Punica III), Roma 2007, pp. 9-30.

2009a Offerte e depositi votivi nel Santuario C3 del Kothon di Mozia nel IV sec. a.C.: S. FORTUNELli - C. MASSERIA (edd.), La ceramica attica dai santuari della Grecia, della Ionia e dell'Italia (Atti del Convegno Internazionale, Perugia 14-17 marzo 2007), Venosa 2009, pp. 703-719. 
2009b Recenti scoperte dell’Università di Roma 'La Sapienza' a Mozia (2002-2006): il Tempio del Kothon, la «Casa del sacello domestico», il «Basamento meridionale» e la Fortezza Occidentale: C. AmPolo (ed.) Immagine e immagini della Sicilia e di altre isole del Mediterraneo antico. Atti delle Seste Giornate Internazionali di Studi sull'area elima e la Sicilia occidentale nel contesto mediterraneo (Erice, 12-16 ottobre 2006), Pisa 2009, pp. 551-559.

2009c Il Tempio del Kothon e il ruolo delle aree sacre nello sviluppo urbano di Mozia dall'VIII al IV secolo a.C.: S. Helas - D. Marzoli (hrsgg.) Phönizisch und punisches Städtewesen. Akten der internationalen Tagung, Rom, 21.-23. Februar 2007 (Iberia Archaeologica 13), Mainz am Rhein 2009, pp. 241-270.

2012 Scavi e restauri dell'Università di Roma «La Sapienza» a Mozia, 2007-2009: il Tempio del Kothon, il Temenos Circolare, il Sacello di Astarte e il Tofet: C. Ampolo (ed.), Sicilia Occidentale" Studi, rassegne e ricerche, Atti delle Settime Giornate Internazionali di Studi sull'area Elima e la Sicilia Occidentale nel contesto mediterraneo (Erice 2009) (Seminari e Convegni 29), Pisa 2012, pp. 207-218.

2013 Mozia: il tofet e la città. Il limite meridionale del santuario e le strutture collegate negli scavi della Sapienza 2010-2011: L.M. MicheTti (ed.), Scienze dell'Antichità 19.1, Roma 2013, pp. 37-53.

2015 Mozia tra IV e III sec. a.C.: nuovi dati dagli scavi della Sapienza: S. TusA - C.A. Buccellato (edd.), La Battaglia delle Egadi. Atti del convegno: Favignana, ex Stabilimento Florio, 20-21 novembre 2015 (Regione Siciliana. Assessorato dei beni culturali e dell'identità siciliana, Dipartimento dei beni culturali e dell'identità siciliana), Palermo 2016, pp. 15-25.

2017 The earliest Tophet: L. Nigro - F. Spagnoli (eds.), Landing on Motya. The earliest Phoenician settlement of the $8^{\text {th }}$ century $B C$ and the creation of a West Phoenician cultural identity in the excavations of Sapienza University of Rome-2012-2016 (Quaderni di Archeologia fenicio-punica/Colour Monograph 04), Roma 2017, pp. 70-73.

2018 La sapienza a Mozia 2010-2016: il primo insediamento fenicio. L'area sacra di Baal e Astarte, il Tofet, la Necropoli, l'Abitato, i nuovi scavi alle Mura - una sintesi: Folia Phoenicia 2 (2018), pp. 253-277.

2020 Nuovi scavi al Tofet di Mozia (2009-2014): il Tempio di Astarte (T6), l'Edificio T5 e il sacello T8: M. Guirguis - S. MuSCuSO - R. Pla OrQín (edd.), Cartagine, il Mediterraneo centro-occidentale e la Sardegna. Società, economia e cultura materiale tra Fenici e autoctoni. Studi in onore di Piero Bartoloni (Le Monografie della SAIC/3), Sassari 2020, pp. 121-146.

NigRO, L. (ed.)

2005 Mozia XI. Il Tempio del Kothon. Rapporto preliminare delle campagne di scavi XXIII e XXVII (2003-2004) condotte congiuntamente con il Servizio Beni Archeologici della Soprintendenza Regionale per i Beni Culturali e Ambientali di Trapani (Quaderni di Archeologia Fenicio-Punica II), Roma 2005.

2011 Mozia-XIII. Zona F. La Porta Ovest e la Fortezza Occidentale. Rapporto preliminare delle campagne di scavi XXIII e XXVII (2003-2007) condotte congiuntamente con il Servizio Beni Archeologici della Soprintendenza Regionale per i Beni Culturali e Ambientali di Trapani (Quaderni di Archeologia Fenicio-Punica VI), Roma 2011. 
NigRO, L. - MELANDRI, I.

2011 La Fortezza Occidentale nel IV sec. a.C.: architettura e ritrovamenti: L. NIGRo (ed.), Mozia-XIII. Zona F. La Porta Ovest e la Fortezza Occidentale. Rapporto preliminare delle campagne di scavi XXIII e XXVII (2003-2007) condotte congiuntamente con il Servizio Beni Archeologici della Soprintendenza Regionale per i Beni Culturali e Ambientali di Trapani (Quaderni di Archeologia Fenicio-Punica VI), Roma 2011, pp. 76102.

NigRo, L. - SPAGNOLI, F.

2004 Il Santuario del "Cappiddazzu”: L. Nigro- G. Rossoni (edd.), «La Sapienza» a Mozia. Quarant'anni di ricerca archeologica, 1964-2004. Catalogo della mostra, Università di Roma «La Sapienza», Facoltà di Scienze Umanistiche, Museo dell'Arte Classica, 27 febbraio - 18 maggio 2004 (Quaderni di Archeologia Fenicio-Punica, Colour Monograph 1), Roma 2004, pp. 56-61.

ORSINGHER, A.

2011 La ceramica punica del IV sec. a.C. dalla Fortezza Occidentale a Mozia: L. NigRo (ed.), Mozia-XIII. La Porta Ovest e la Fortezza Occidentale. Rapporto preliminare delle campagne di scavo XXIII-XXVII (2003-2007) condotte congiuntamente con il Servizio Beni Archeologici della Soprintendenza Regionale per i Beni Culturali e Ambientali di Trapani (Quaderni di Archeologia Fenicio-Punica VI), Roma 2011, pp. 112-128.

2013a The Hellenisation of the Punic World: a view from the Tophet: L. BombardierI - A D'Agostino - G. GuARDUCCI - V. ORSI - S. VAlENTINI (eds.), SOMA 2012 Identity and Connectivity. Proceedings of the 16 Symposium on Mediterranean Archeology, Florence, Italy, 1 - 3 March 2012 (BAR International Series 2581, vol. II), Oxford 2013, pp. 693701.

2013b La ceramica di impasto a Mozia tra cultura fenicia e tradizione indigena: L. GIRÓN Anguiozar - M. LAZARich GonZÁlez - M. DA CONCEIÇAO Lopes (eds.), Actas del I Congreso Internacional sobre Estudios Ceràmicos, Homenaje a la Dra. Mercedes Vegas (Cádiz del 1 al 5 noviembre de 2010) (Actas, Historia y Arte), Cádiz 2013, pp. 757-790.

2015 Vessels in Tophet sanctuaries: the archaic evidence and the levantine connection: A. M. Maila Afeiche (ed.) Proceedings of the International Symposium Beirut 2012 "Cult and Ritual on the Levantine Coast and its impact on the Eastern Mediterranean Realm" (Bulletin d'Archéologie et d'Architecture Libanaises, Hors-Serie X), Beirut 2015, pp. 561-590.

2016 The ceramic repertoire of Motya: origins and development between the 8 and 6 centuries B.C.: F. SCHON - H. TOPFER (hrsgg.) Karthago Dialoge. Karthago und der punische Mittelmeerraum- Kulturkontakte und Kulturtransfers im.1 Jahrtausend vor Christus (Ressourcen Kulturen 2), Tubingen 2016, pp. 283-312.

PANVINI, R.

2010 Le rayonnement des sanctuaires grecs de Géla sur l'arrière-pays sicane: J. DE LA GENIERE - A. VAUCHEZ - J. LeClANT (éd.), Les sanctuaires et leur rayonnement dans le monde méditerranéen de L'Antiquité à l'époque moderne, Actes du 20e colloque de la Villa Kérylos à Beaulieu-sur-Mer les 9 \& 10 octobre 2009 (Cahiers de la Villa «Kérylos»21), Parigi 2010, pp. 39-63.

PARISI, V.

2017 I depositi votivi negli spazi del rito. Analisi dei contesti per un'archeologia della pratica cultuale nel mondo siceliota e magnogreco (Supplementi e monografie della rivista Archeologia classica 14, n.s. 11), Roma 2017. 
Rocco, G.

2004 Zona C. Il Kothon. La ceramica greca e coloniale: L. NIGRO (ed.), Mozia - X. Rapporto preliminare della XXII campagna di scavi - 2002 condotta congiuntamente con il Servizio Beni Archeologici della Soprintendenza Regionale per i Beni Culturali e Ambientali di Trapani (Quaderni di Archeologia Fenicio-Punica I), Roma 2004, pp. 87-89.

2007 Le ceramiche attiche e coloniali dipinte e a vernice nera: L. NIGRO (ed.), Mozia - XII. Zona D. La "Casa del sacello domestico", il "Basamento meridionale" e il Sondaggio stratigrafico I. Rapporto preliminare delle campagne di scavi XIII e XXIV (2003-2004) condotte congiuntamente con il Servizio Beni Archeologici della Soprintendenza Regionale per i Beni Culturali e Ambientali di Trapani (Quaderni di Archeologia FenicioPunica II), Roma 2007, pp. 56-58.

2011 Le ceramiche greche e coloniali a figure nere, rosse e a vernice nera: L. NigRo (ed.), Mozia-XIII. La Porta Ovest e la Fortezza Occidentale. Rapporto preliminare delle campagne di scavo XXIII-XXVII (2003-2007) condotte congiuntamente con il Servizio Beni Archeologici della Soprintendenza Regionale per i Beni Culturali e Ambientali di SPAGNOLI, F. Trapani (Quaderni di Archeologia Fenicio-Punica VI), Roma 2011, pp. 108-111.

2012 I rinvenimenti ceramici dal pozzo sacro P.2927: L. NigRo - F. SPAGNOLi (edd.), Alle sorgenti del Kothon. Il rito a Mozia nell'Area sacra di Baal 'Addir- Poseidon. Lo scavo dei pozzi sacri nel Settore C Sud-Ovest (2006-2011). (Quaderni di Archeologia FenicioPunica, Colour Monograph 02), Roma 2012, pp. 38-48.

SPANÒ GIAMMELlaro, A.

2000 Scavi nella Zona K di Mozia. L'ottava campagna di scavo (Maggio-Luglio 1994): M.E. Aubet - M. BARthélemy (eds.), Actas del IV Congreco International de Estudios Fenicios y Púnicos (Cádiz, 2 al 6 de Octubre de 1995), Cádiz 2000, pp. 1377-1395.

SPARKES, B.A. - TALCOTT, L.

1970 Black and Plain Pottery of the 6, 5 and 4 Centuries B.C. (Athenian Agora XII), Princeton 1970.

TARDO, V.

1997 Materiali dalla necropoli punica di Solunto: studi preliminari. Ceramica di tradizione e di importazione greca: C. Greco - F. Spatafora - S. VAsSAllo (edd.), Archeologia e territorio (Beni Culturali Palermo), Palermo 1997, pp. 75-93.

2005 Ceramica di importazione e tradizione greca da Solunto; A. SPANò Giammellaro (ed.), Atti del V Congresso Internazionale di Studi Fenici e Punica (Marsala-Palermo 2-8 ottobre 2000), Vol. I, Palermo 2005, pp. 677-687.

TотI, M.P.

2002a Lucerne: M.L. FAMÀ (ed.), Mozia. Gli scavi nella zona A dell'abitato (Collana di archeologia del centro internazionale di Studi Fenici, Punici, Romani, Comune di Marsala 1), Bari 2002, pp. 305-354.

2002b Alcune considerazioni sulla produzione vascolare di officine dell'isola di Mozia (TP): M.G. Amadasi Guzzo - M. Liverani - P. Matthiae (edd.) Da Pyrgi a Mozia. Studi sull'archeologia del Mediterraneo in memoria di Antonia Ciasca (Quaderni di Vicino Oriente 3/2), Roma 2002, pp. 555-565.

TRONCHETTI, C.

2016 La necropoli di Tuvixeddu: la ceramica di importazione: Sardinia, Corsica et Baleares Antiquae XIV (2016), pp. 83-106.

2020 La ceramica attica di IV sec. a.C. in Sardegna e oltre: M. GuIRGuIS - S. Muscuso - R. Pla ORQuín (edd.), Cartagine, il Mediterraneo centro-occidentale e la Sardegna. Società, 
TUSA, V.

1973 Il luogo di arsione. Lo scavo del 1971: A. CiASCA - V. TuSA - M.L. UBERTI (edd.) MoziaVIII. Rapporto preliminare della Missione congiunta con la Soprintendenza alle Antichità della Sicilia Occidentale (Studi Semitici 45), Roma 1973, pp. 35-56.1978 La Necropoli arcaica e adiacenze: A. Ciasca - G. CoACCi Polselli - N. CuOMO Di CAPrio - M.G. Guzzo Amadasi - G. Matthiae Scandone - V. Tusa - A. Tusa Cutroni - M.L. Uberti (edd.) Mozia-IX. Rapporto preliminare della Missione congiunta con la Soprintendenza alle Antichità della Sicilia Occidentale (Studi Semitici 50), Roma 1978, pp. 7-90.

VASSALLO, S.

2016 Sulla presenza del guttus nelle sepolture infantili delle necropoli imeresi: dati preliminari: E. LATTANZI - R. SPADEA (edd.), Se cerchi la tua strada verso Itaca... Omaggio a Lina Di Stefano, Roma 2016, pp. 49-57. 


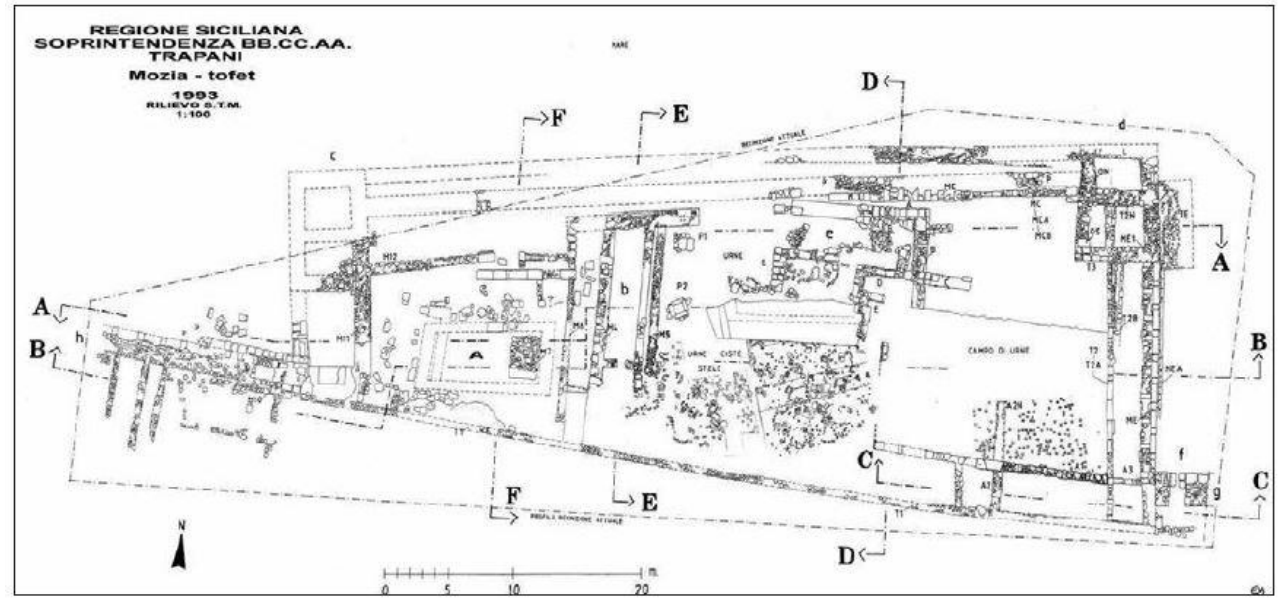

Fig. 1 - Planimetria generale del Tofet di Mozia (da Giglio 2012, fig. 326).

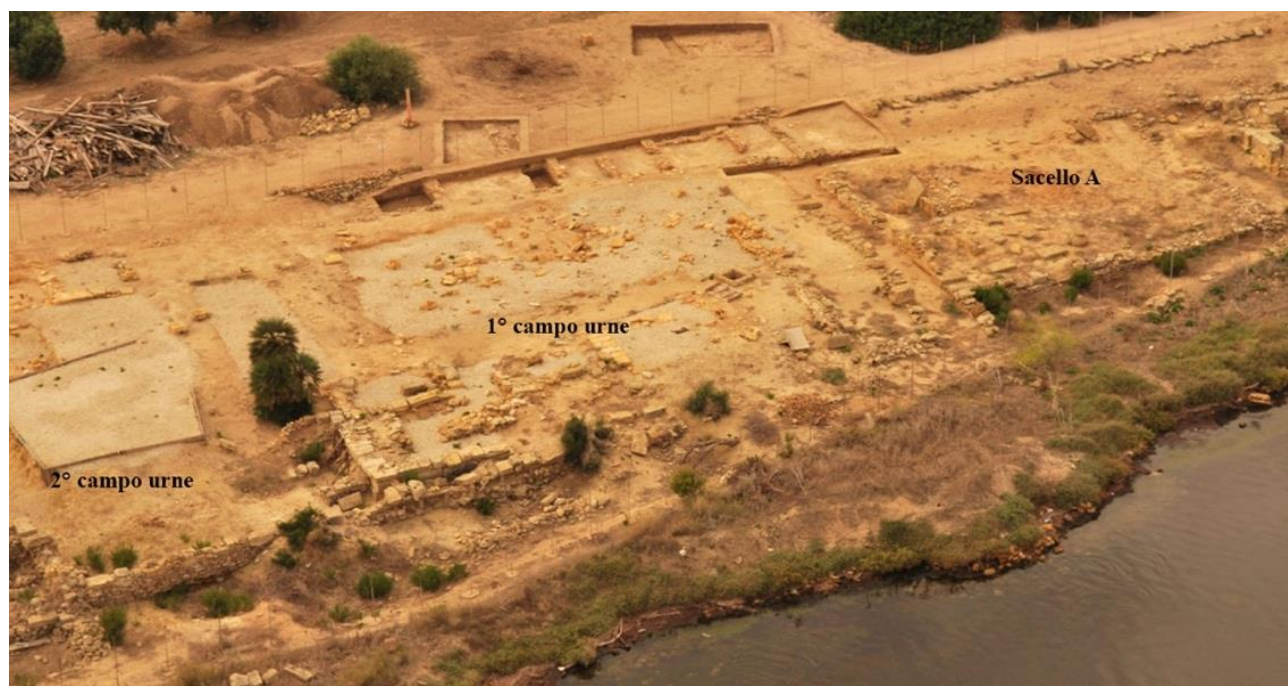

Fig. 2 - Veduta area del Tofet da nord. 


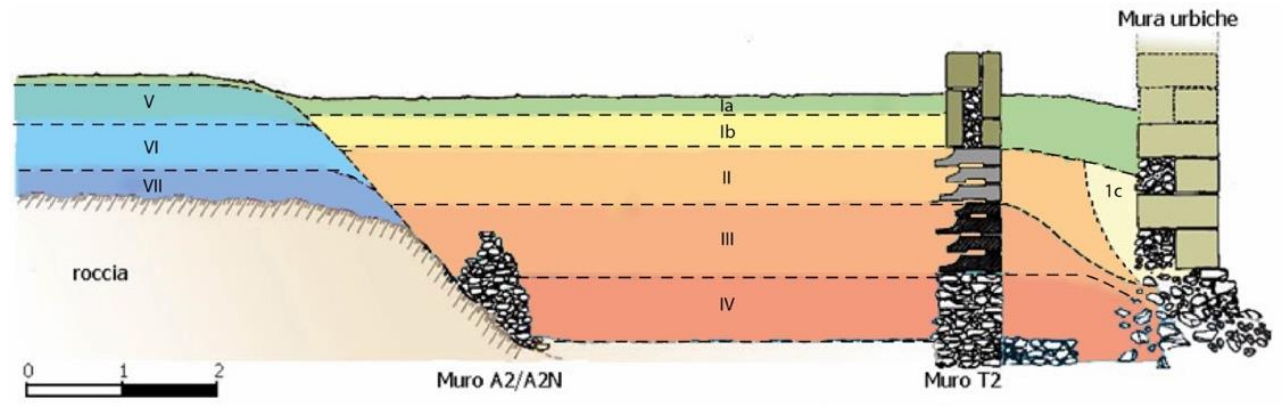

Fig. 3 - Rielaborazione della sezione stratigrafica schematica E-O del campo di urne (da Ciasca 1992, 155, fig. 7).

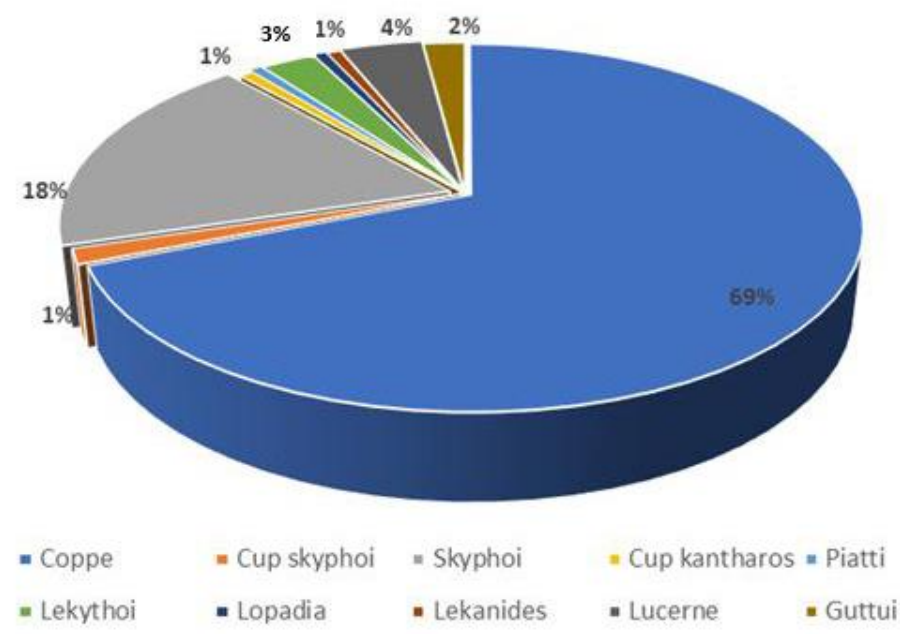

Fig. 4 - Composizione del repertorio ceramico a vernice nera dal Tofet.

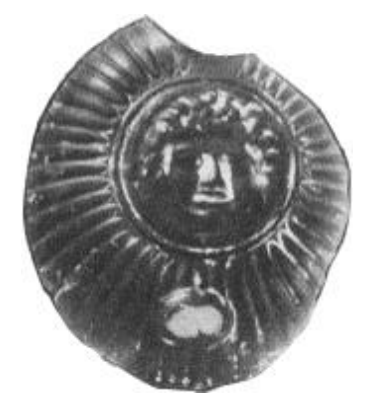

Fig. 5 - Guttus con gorgoneion dall'ultimo strato deposizionale del Tofet (da Ciasca 1966, tav. LII). 

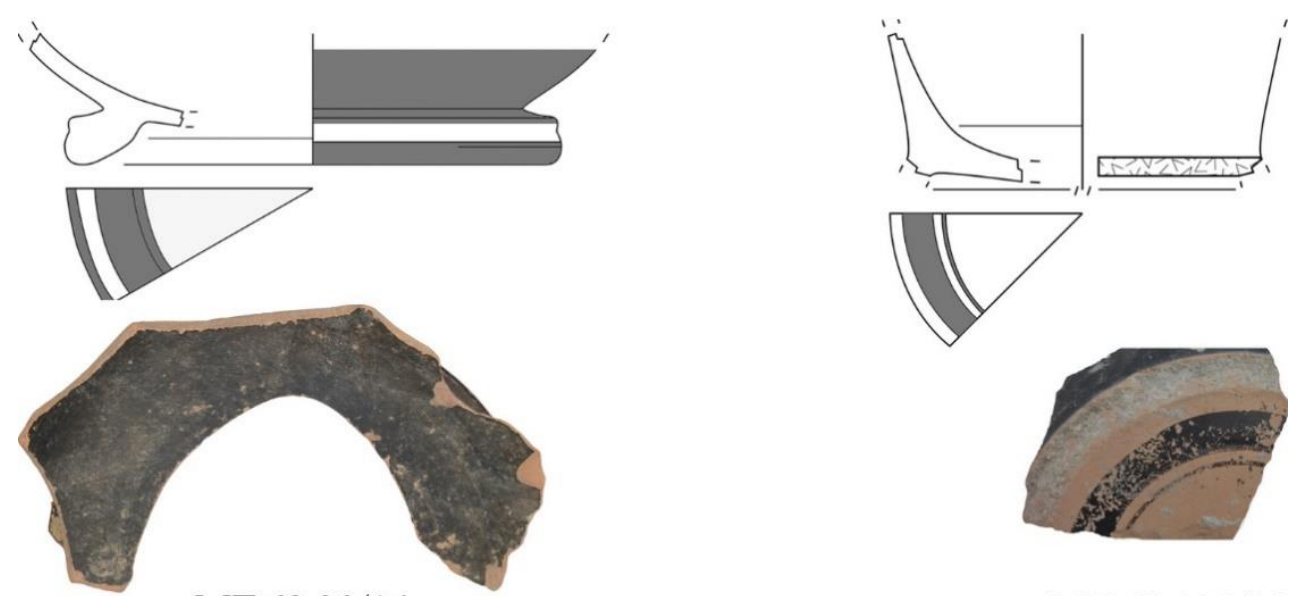

MT.68.30/14
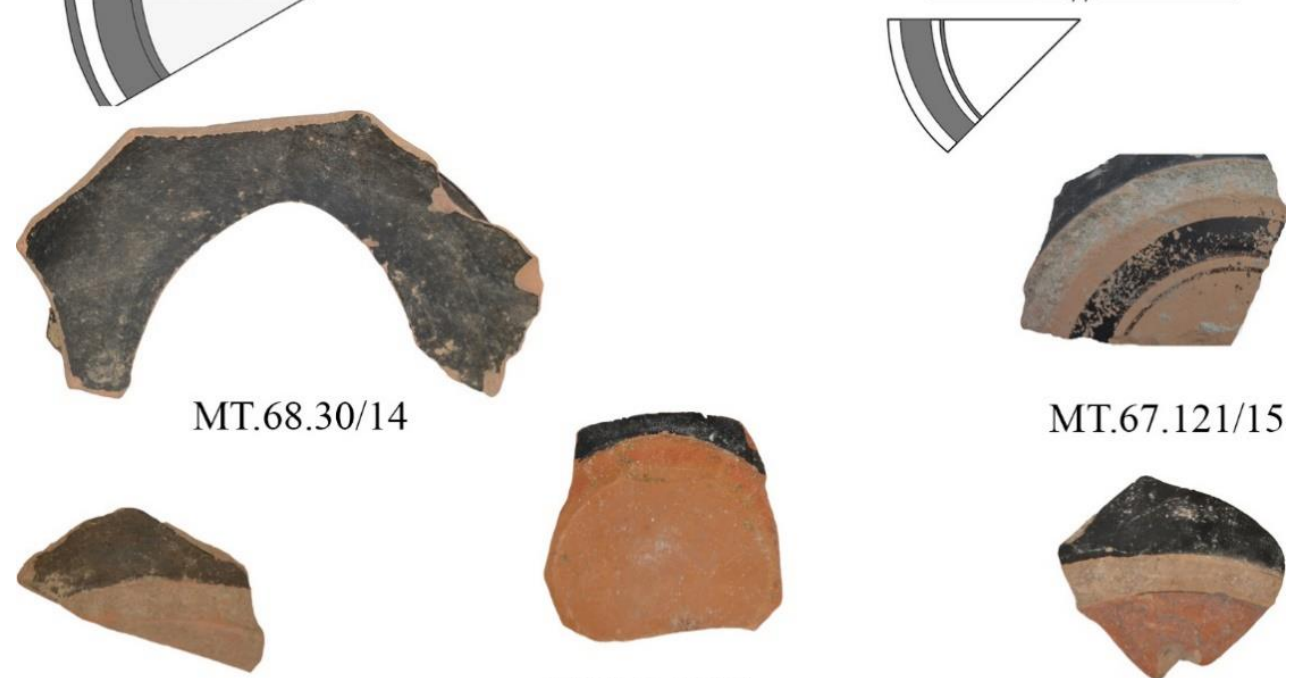

MT.68.12/11

MT.73.19/7

MT.67.121/15

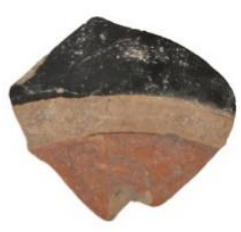

MT.68.9/13

Fig. 6 - La coppa stemless forata per libare e gli skyphoi defunzionalizzati.

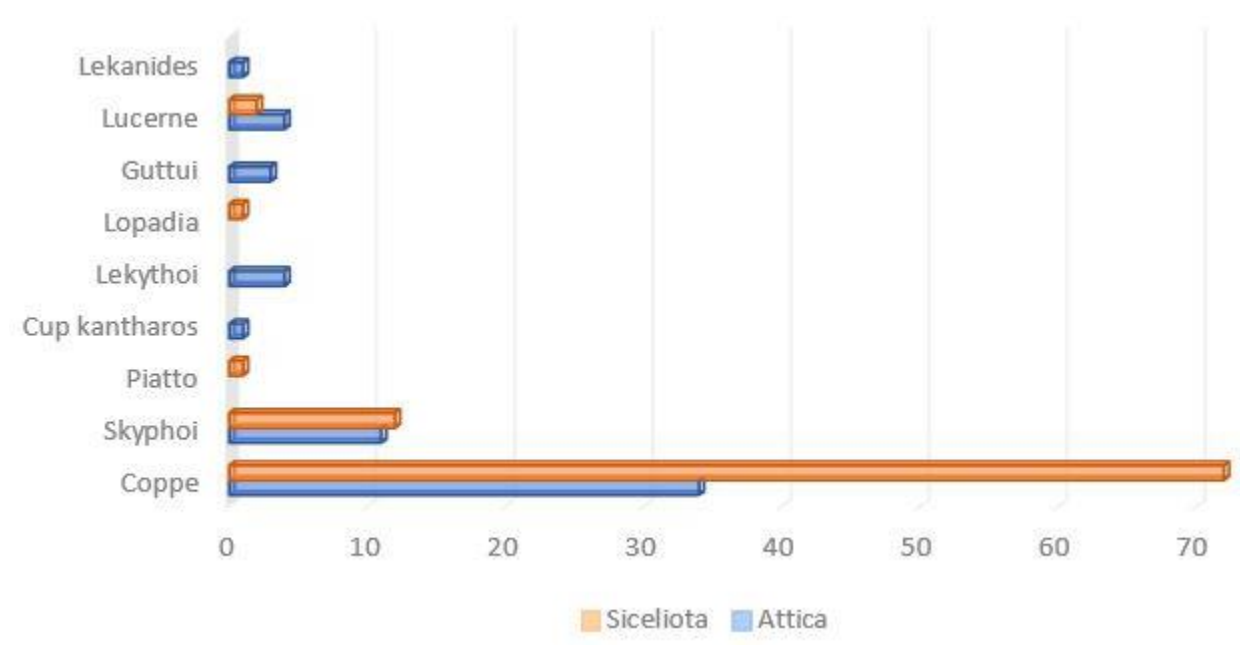

Fig. 7 - Le forme di importazione attica e di imitazione siceliota dal Tofet. 

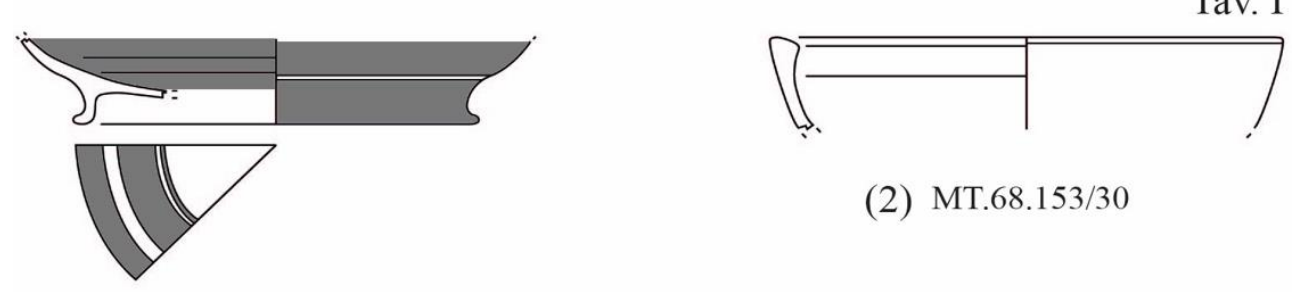

(2) MT.68.153/30

(1) MT.68.73/14

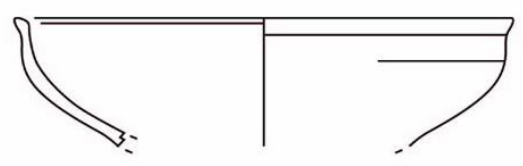

(3) MT.67.158/7

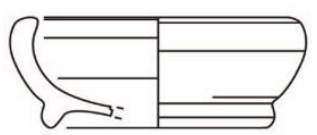

(4) MT.70.31/4

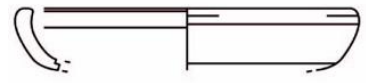

(7) MT.68.28/1

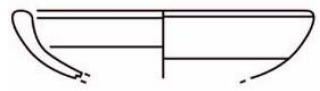

(10) MT.68.34/16

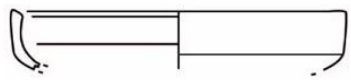

(13) MT.68.153/42

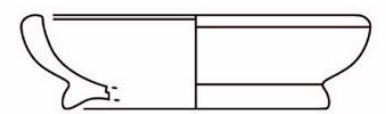

(5) MT.65.1541

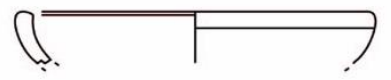

(8) MT.67.7/23

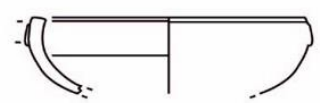

(11) MT.68.112/41

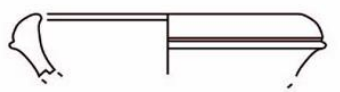

(14) MT.67.10/10

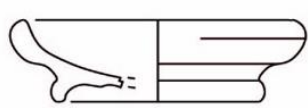

(6) MT.66.993

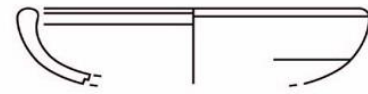

(9) MT.65.182

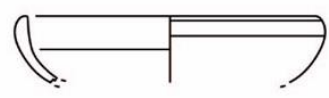

(12) MT.68.153/49

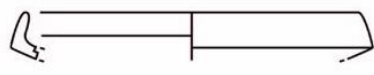

(15) MT.70.53/2

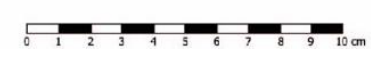




\begin{tabular}{|c|c|c|c|c|c|c|c|}
\hline N. & Forma & Produzione & Colore & $\emptyset \mathrm{cm}$ & \begin{tabular}{|c|} 
Datazione \\
a.C.
\end{tabular} & Bibliografia & $\begin{array}{c}\text { N. tipi } \\
\text { analoghi }\end{array}$ \\
\hline 1 & Bolsal & Attica & $\begin{array}{c}7.5 \text { YR7/6 } \\
\text { Reddish Yellow }\end{array}$ & 14 & 400 & $\begin{array}{c}\text { Sparkes - Talcott 1970, fig. 6, } \\
\text { n. } 554\end{array}$ & 5 \\
\hline 2 & $\begin{array}{l}\text { Coppa broad } \\
\text { rim }\end{array}$ & Attica & $\begin{array}{c}\text { 5YR8/6 } \\
\text { Reddish Yellow }\end{array}$ & 14 & $400-350$ & $\begin{array}{c}\text { Sparkes - Talcott 1970, 292, } \\
\text { fig. } 8, n .783\end{array}$ & 3 \\
\hline 3 & $\begin{array}{c}\text { Coppa } \\
\text { outturned rim }\end{array}$ & Attica & $\begin{array}{c}\text { 5YR7/6 } \\
\text { Reddish Yellow }\end{array}$ & 14 & $350-300$ & $\begin{array}{c}\text { Sparkes - Talcott 1970, fig. 8, } \\
\text { n. } 802 .\end{array}$ & 3 \\
\hline 4 & $\begin{array}{c}\text { Coppa } \\
\text { incurving rim }\end{array}$ & Attica & $\begin{array}{c}\text { 2.5YR6/1 } \\
\text { Gray }\end{array}$ & $\begin{array}{l}9 \\
6\end{array}$ & $400-375$ & $\begin{array}{c}\text { Sparkes - Talcott 1970, fig. 9, } \\
\text { n. } 861 .\end{array}$ & 3 \\
\hline 5 & \begin{tabular}{|c|} 
Coppa \\
incurving rim \\
\end{tabular} & Attica & $\begin{array}{l}10 \mathrm{Y} 7 / 8 \\
\text { Red }\end{array}$ & $\begin{array}{l}8 \\
7 \\
\end{array}$ & $400-375$ & Michelini 2002, 181, n. 56. & 9 \\
\hline 6 & \begin{tabular}{|c|} 
Coppa \\
incurving rim \\
\end{tabular} & Attica & $\begin{array}{c}\text { 5YR7/6 } \\
\text { Reddish Yellow }\end{array}$ & $\begin{array}{l}9 \\
6 \\
\end{array}$ & $400-375$ & Michelini 2002, 181, n. 60. & 1 \\
\hline 7 & \begin{tabular}{|c|} 
Coppa \\
incurving rim \\
\end{tabular} & Attica & $\begin{array}{l}2.5 Y R 7 / 6 \\
\text { Light Red }\end{array}$ & 8 & $410-390$ & $\begin{array}{c}\text { Sparkes - Talcott 1970, fig. 8, } \\
\text { n. } 825\end{array}$ & 4 \\
\hline 8 & \begin{tabular}{|c|} 
Coppa \\
incurving rim \\
\end{tabular} & Attica & $\begin{array}{c}10 Y 7 / 8 \\
\text { Red }\end{array}$ & 10 & $410-390$ & $\begin{array}{c}\text { Sparkes - Talcott 1970, fig. 8, } \\
\text { n. } 876 \\
\end{array}$ & 5 \\
\hline 9 & \begin{tabular}{|c|} 
Coppa \\
incurving rim \\
\end{tabular} & Attica & $\begin{array}{c}\text { 5YR7/6 } \\
\text { Reddish Yellow }\end{array}$ & 10 & $400-375$ & Michelini 2002, 180, n. 48 & 11 \\
\hline 10 & \begin{tabular}{|c|} 
Coppa \\
incurving rim \\
\end{tabular} & Attica & $\begin{array}{l}7.5 \mathrm{YR} 8 / 4 \\
\text { Pink }\end{array}$ & 8 & $400-375$ & $\begin{array}{c}\text { Sparkes - Talcott 1970, fig. 9, } \\
\text { n. } 882\end{array}$ & 1 \\
\hline 11 & \begin{tabular}{|c|} 
Coppa \\
incurving rim \\
\end{tabular} & Attica & $\begin{array}{c}\text { 5YR7/6 } \\
\text { Reddish Yellow }\end{array}$ & 8 & $400-375$ & Michelini 2002, 178, n. 41 & 1 \\
\hline 12 & $\begin{array}{c}\text { Coppa } \\
\text { incurving rim }\end{array}$ & Attica & $\begin{array}{c}\text { 5YR8/6 } \\
\text { Reddish Yellow }\end{array}$ & 8 & $400-375$ & $\begin{array}{c}\text { Sparkes }- \text { Talcott 1970, fig. 8, } \\
\text { n. } 828\end{array}$ & 1 \\
\hline 13 & $\begin{array}{l}\text { Coppa one } \\
\text { handler }\end{array}$ & Attica & $\begin{array}{c}\text { 7.5YR7/2 } \\
\text { Pinkish Gray }\end{array}$ & 8 & $400-350$ & $\begin{array}{c}\text { Sparkes - Talcott 1970, fig. 8, } \\
\text { n. } 757\end{array}$ & 1 \\
\hline 14 & \begin{tabular}{|c|}
$\begin{array}{c}\text { Coppa shallow } \\
\text { walls }\end{array}$ \\
\end{tabular} & Attica & $\begin{array}{c}5 Y R 7 / 6 \\
\text { Reddish Yellow }\end{array}$ & 8 & 390 & $\begin{array}{c}\text { Sparkes - Talcott 1970, fig. 8, } \\
\text { n. } 821\end{array}$ & 1 \\
\hline 15 & Coppa carenata & Attica & $\begin{array}{c}\text { 5YR6/6 } \\
\text { Reddish Yellow }\end{array}$ & 10 & 350 & $\begin{array}{c}\text { Sparkes - Talcott 1970, 295, } \\
\text { fig. } 8, n .829\end{array}$ & 1 \\
\hline
\end{tabular}




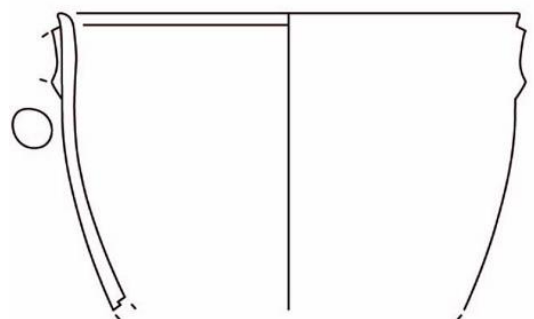

(16) MT.71.85/33

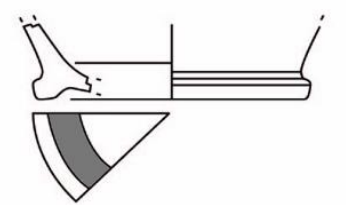

(17) MT.68.112/8
Tav. II

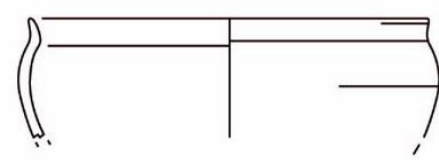

(18) MT.66.354

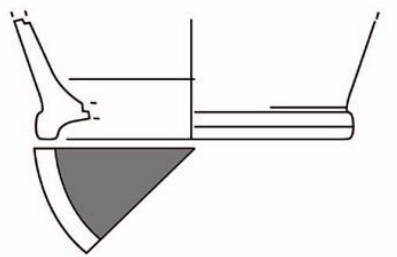

(19) MT.68.112/13

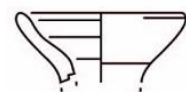

(20) MT.67.165/40

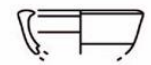

(21) MT.68.84/26

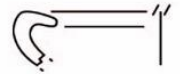

(23) MT.65.594

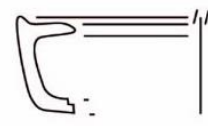

(24) MT.72.32/1

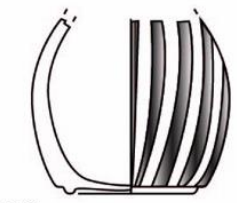

(22) MT.73.2/1

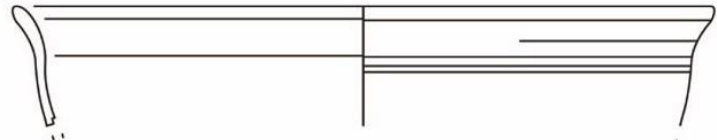

(25) MT.70.31/3

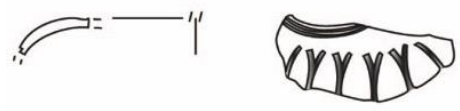

(26) MT.66.1284

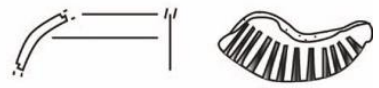

(27) MT.66.595 


\begin{tabular}{|c|c|c|c|c|c|c|c|}
\hline N. & Forma & Produzione & Colore & $\varnothing \mathrm{cm}$ & $\begin{array}{c}\text { Datazione } \\
\text { a.C. }\end{array}$ & Bibliografia & $\begin{array}{c}\text { N. tipi } \\
\text { analoghi }\end{array}$ \\
\hline 16 & Skyphos & Attica & $\begin{array}{c}7.5 \text { YR6/6 } \\
\text { Reddish Yellow }\end{array}$ & 13 & $400-375$ & $\begin{array}{c}\text { Sparkes - Talcott 1970, 260, } \\
\text { fig. } 4 \text {, n. } 349\end{array}$ & 1 \\
\hline 17 & Skyphos & Attica & $\begin{array}{l}\text { 7.5YR7/3 } \\
\text { Pink }\end{array}$ & 9 & $400-375$ & $\begin{array}{c}\text { Sparkes - Talcott 1970, 260, } \\
\text { fig. } 4, \text { n. } 349\end{array}$ & 6 \\
\hline 18 & Skyphos & Attica & $\begin{array}{c}\text { 5YR7/6 } \\
\text { Reddish Yellow }\end{array}$ & 12 & $350-330$ & $\begin{array}{l}\text { Sparkes - Talcott 1970, 260, } \\
\text { fig. } 4, n .352\end{array}$ & 1 \\
\hline 19 & Skyphos & Attica & $\begin{array}{l}7.5 Y R 7 / 3 \\
\text { Pink }\end{array}$ & 9 & $350-330$ & $\begin{array}{l}\text { Sparkes - Talcott 1970, 260, } \\
\quad \text { fig. } 4, n .352\end{array}$ & 2 \\
\hline 20 & Lekythos & Attica & $\begin{array}{l}5 Y \text { YR7/3 } \\
\text { Pink } \\
\end{array}$ & 5 & $410-390$ & $\begin{array}{c}\text { Sparkes - Talcott 1970, 313, } \\
\text { fig. } 11, n .1099 \\
\end{array}$ & 1 \\
\hline 21 & Lekythos & Attica & $\begin{array}{c}\text { 5YR5/6 } \\
\text { Reddish Yellow }\end{array}$ & 3 & $375-325$ & $\begin{array}{c}\text { Sparkes - Talcott 1970, 315, } \\
\text { fig. } 11, n .1123\end{array}$ & 1 \\
\hline 22 & Lekythos & Attica & 7.5YR7/4 Pink & 4 & $375-325$ & $\begin{array}{c}\text { Sparkes - Talcott 1970, 315, } \\
\text { pl. } 28, \text { n. } 1129\end{array}$ & 1 \\
\hline 23 & $\begin{array}{c}\text { Lucerna tipo } \\
23 \mathrm{~A}\end{array}$ & Attica & $\begin{array}{c}\text { 7.5YR7/6 } \\
\text { Reddish Yellow }\end{array}$ & I & 410-375 & $\begin{array}{c}\text { Howland 1958, 65, pl. 9.37, } \\
\text { n. } 255\end{array}$ & 2 \\
\hline 24 & $\begin{array}{c}\text { Lucerna tipo } \\
\text { 24B } \\
\end{array}$ & Attica & $\begin{array}{c}5 Y R 7 / 6 \\
\text { Reddish Yellow } \\
\end{array}$ & I & $410-375$ & $\begin{array}{c}\text { Howland 1958, 57, pl. 7.36, } \\
\text { n. } 216\end{array}$ & 2 \\
\hline 25 & Kantharos & Attica & $\begin{array}{c}\text { 5YR6/6 } \\
\text { Reddish Yellow }\end{array}$ & 18 & $400-375$ & $\begin{array}{c}\text { Sparkes - Talcott 1970, 279, } \\
\text { fig. } 6, \text { n. } 617\end{array}$ & 1 \\
\hline 26 & $\begin{array}{c}\text { Guttus askos } \\
\text { type }\end{array}$ & Attico & $\begin{array}{l}5 Y \text { YR7/3 } \\
\text { Pink }\end{array}$ & l & $400-375$ & Di Stefano 2009, 39, fig. 54. & 1 \\
\hline 27 & $\begin{array}{c}\text { Guttus askos } \\
\text { type }\end{array}$ & Attico & $\begin{array}{l}5 \text { YR7/3 } \\
\text { Pink } \\
\end{array}$ & l & $400-375$ & Di Stefano 2009, 39, fig. 54. & 1 \\
\hline
\end{tabular}




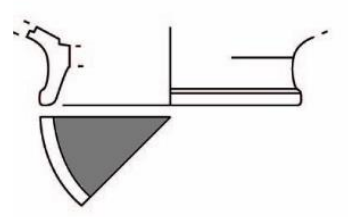

(28) MT.71.118/1

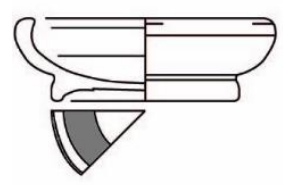

(31) MT.70.34/1

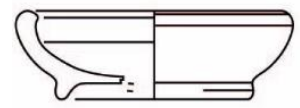

(34) MT.70.19/1

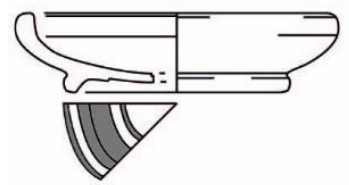

(37) MT.71.35/34

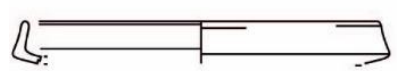

(40) MT.65.1169

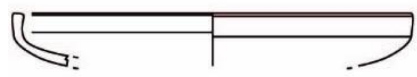

(41) MT.72.58/5

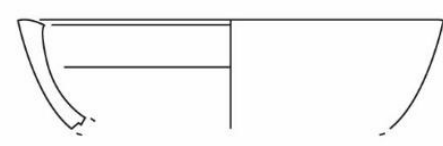

(29) MT.65.n.s

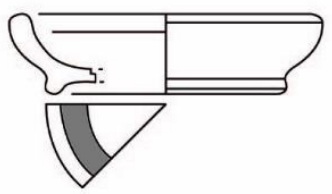

(32) MT.67.108/17

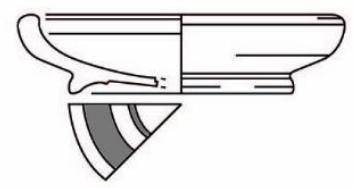

(35) MT.68.73/13

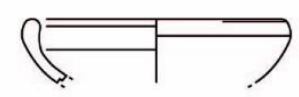

(38) MT.65.519
Tav. III

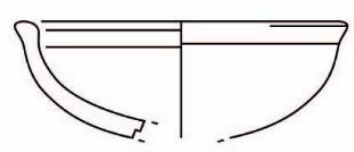

(30) MT.65.886

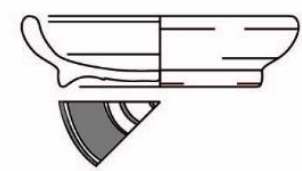

(33) MT.70.107/1

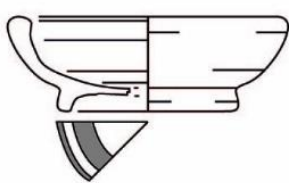

(36) MT.70.97

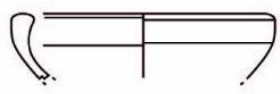

(39) MT.67.160/20

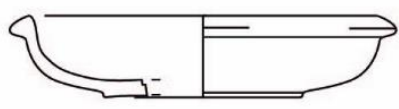

(42) MT.67.197/7

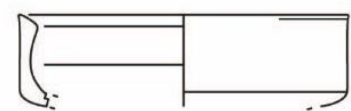

(43) MT.71.123/8

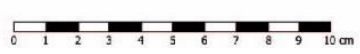




\begin{tabular}{|c|c|c|c|c|c|c|c|}
\hline N. & Forma & Produzione & Colore & $\varnothing \mathrm{cm}$ & \begin{tabular}{|c|} 
Datazione \\
a.C.
\end{tabular} & Bibliografia & $\begin{array}{c}\text { N. tipi } \\
\text { analoghi }\end{array}$ \\
\hline 28 & Coppa stemless & Coloniale & $\begin{array}{c}7.5 \text { YR7/6 } \\
\text { Reddish Yellow } \\
\end{array}$ & 6 & $410-390$ & $\begin{array}{c}\text { Sparkes - Talcott 1970, fig. 5, } \\
\text { n. } 515\end{array}$ & 2 \\
\hline 29 & $\begin{array}{l}\text { Coppa broad } \\
\text { rim }\end{array}$ & Coloniale & $\begin{array}{c}\text { 7.5YR7/6 } \\
\text { Reddish Yellow }\end{array}$ & 12 & $375-350$ & $\begin{array}{c}\text { Morel 1981, 245, pl.85, } \\
2985 \mathrm{c} 1\end{array}$ & 1 \\
\hline 30 & \begin{tabular}{|c|} 
Coppa \\
outturned rim \\
\end{tabular} & Coloniale & $\begin{array}{l}5 Y R 7 / 4 \\
\text { Pink }\end{array}$ & 10 & $350-300$ & $\begin{array}{c}\text { Morel 1981, 204, pl.65, } \\
2672 b\end{array}$ & 1 \\
\hline 31 & $\begin{array}{c}\text { Coppetta } \\
\text { incurving rim }\end{array}$ & Coloniale & $\begin{array}{l}2.5 Y R 6 / 6 \\
\text { Light Red }\end{array}$ & $\begin{array}{l}7 \\
6\end{array}$ & $400-375$ & Michelini 2002, 182, n. 65 & 9 \\
\hline 32 & \begin{tabular}{|c|} 
Coppetta \\
incurving rim \\
\end{tabular} & Coloniale & $\begin{array}{c}7.5 \text { YR7/6 } \\
\text { Reddish Yellow } \\
\end{array}$ & $\begin{array}{l}9 \\
7 \\
\end{array}$ & $400-375$ & Michelini 2002, 181, n. 59 & 3 \\
\hline 33 & \begin{tabular}{|c|} 
Coppetta \\
incurving rim \\
\end{tabular} & Coloniale & $\begin{array}{c}7.5 \mathrm{YR} 5 / 1 \\
\text { Gray }\end{array}$ & $\begin{array}{c}7,5 \\
6 \\
\end{array}$ & $400-375$ & Michelini 2002, 181, n. 60 & 2 \\
\hline 34 & $\begin{array}{c}\text { Coppetta } \\
\text { incurving rim } \\
\end{array}$ & Coloniale & $\begin{array}{c}\text { 7.5YR7/6 } \\
\text { Reddish Yellow }\end{array}$ & $\begin{array}{l}7 \\
6 \\
\end{array}$ & $400-375$ & Michelini 2002, 181, n.56 & 8 \\
\hline 35 & $\begin{array}{c}\text { Coppetta } \\
\text { incurving rim } \\
\end{array}$ & Coloniale & $\begin{array}{c}\text { 7.5YR7/6 } \\
\text { Reddish Yellow } \\
\end{array}$ & $\begin{array}{c}12 \\
8\end{array}$ & $400-375$ & Michelini 2002, 181, n. 54 & 6 \\
\hline 36 & $\begin{array}{c}\text { Coppetta } \\
\text { incurving rim }\end{array}$ & Coloniale & $\begin{array}{c}\text { 5YR6/6 } \\
\text { Reddish Yellow }\end{array}$ & $\begin{array}{l}8,3 \\
5,5\end{array}$ & $400-375$ & Michelini 2002, 181, n. 59 & 8 \\
\hline 37 & $\begin{array}{c}\text { Coppetta } \\
\text { incurving rim }\end{array}$ & Coloniale & $\begin{array}{c}10 \mathrm{YR} 7 / 4 \\
\text { Very Pale Brown }\end{array}$ & $\begin{array}{l}10 \\
6,5\end{array}$ & $400-375$ & Michelini 2002, 182, n. 65 & 10 \\
\hline 38 & $\begin{array}{c}\text { Coppetta } \\
\text { incurving rim }\end{array}$ & Coloniale & $\begin{array}{c}2.5 \text { YR7/4 } \\
\text { Light Reddish } \\
\text { Brown } \\
\end{array}$ & 7 & $400-350$ & Michelini 2002, 182, n. 44 & 7 \\
\hline 39 & $\begin{array}{c}\text { Coppetta } \\
\text { incurving rim }\end{array}$ & Coloniale & $\begin{array}{l}7.5 \mathrm{YR} 8 / 4 \\
\text { Pink }\end{array}$ & 8 & $400-350$ & Michelini 2002, 181, n. 57 & 2 \\
\hline 40 & Coppa carenata & Coloniale & $\begin{array}{l}\text { 7.5YR5/1 } \\
\text { Gray }\end{array}$ & 11 & $350-325$ & $\begin{array}{c}\text { Morel 1981, 216, pl. 69, } \\
2744 \mathrm{~b} \mathrm{1}\end{array}$ & 1 \\
\hline 41 & Coppa carenata & Coloniale & $\begin{array}{c}7.5 \text { YR7/6 } \\
\text { Reddish Yellow }\end{array}$ & 11 & $350-325$ & $\begin{array}{c}\text { Morel 1981,230, pl. 77, } \\
\text { 2831a 2 }\end{array}$ & 1 \\
\hline 42 & Coppa & Coloniale & $\begin{array}{l}2.5 \text { YR7/2 } \\
\text { Pale Red }\end{array}$ & 10 & $350-300$ & $\begin{array}{c}\text { Morel 1981, 118, tav. 20, } n . \\
1514 b\end{array}$ & 1 \\
\hline 43 & Coppa & Coloniale & $\begin{array}{c}\text { 7.5YR8/6 } \\
\text { Reddish Yellow }\end{array}$ & 10 & $350-300$ & $\begin{array}{c}\text { Sparkes - Talcott 1970, fig. 8, } \\
n .757\end{array}$ & 1 \\
\hline
\end{tabular}




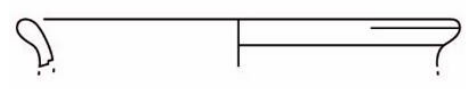

(44) MT.67.115/7

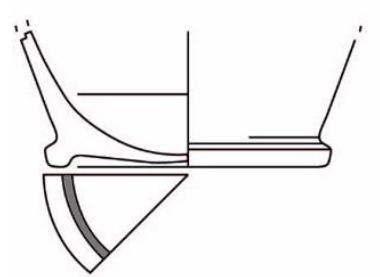

(45) MT.70.100/7

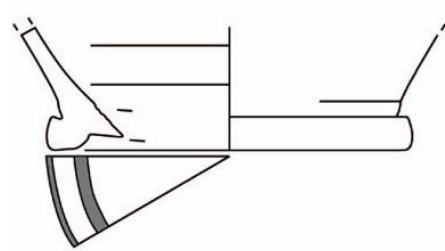

(46) MT.68.73/10

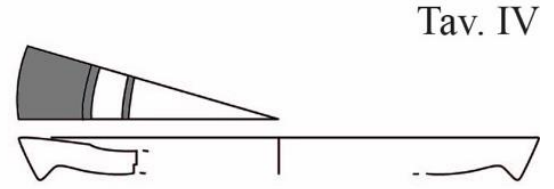

(47) MT.65.102

(48) MT.65.1659

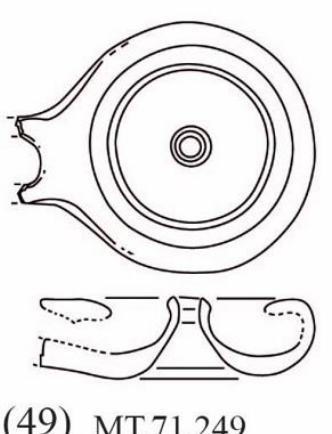

(49) MT.71.249

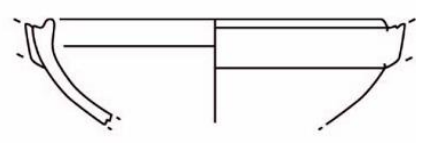

(51) MT.68.16/10 


\begin{tabular}{|c|c|l|c|c|c|c|c|}
\hline N. & Forma & Produzione & Colore & $\varnothing \mathrm{cm}$ & $\begin{array}{c}\text { Datazione } \\
\text { a.C. }\end{array}$ & Bibliografia & $\begin{array}{c}\text { N. tipi } \\
\text { analogh } \\
\text { i }\end{array}$ \\
\hline 44 & Skyphos & Coloniale & $\begin{array}{c}5 \text { YR6/4 } \\
\text { Light Reddish } \\
\text { Brown }\end{array}$ & 12 & $375-350$ & Michelini 2002, 176, n. 31 & 1 \\
\hline 45 & Skyphos & Coloniale & $\begin{array}{c}7.5 \text { YR7/6 } \\
\text { Reddish Yellow }\end{array}$ & 8 & $375-350$ & Michelini 2002, 186, n. 97 & 7 \\
\hline 46 & Skyphos & Coloniale & $\begin{array}{c}7.5 \text { YR7/4 } \\
\text { Pink }\end{array}$ & 10 & $375-350$ & Michelini 2002, 186, n. & 3 \\
\hline 47 & Piatto & Coloniale & $\begin{array}{c}2.5 \text { YR7/6 } \\
\text { Light Red }\end{array}$ & 16 & $375-350$ & $\begin{array}{c}\text { Morel 1981, 87, pl. 4, serie } \\
1128 a\end{array}$ & 1 \\
\hline 48 & $\begin{array}{c}\text { Lucerna tipo } \\
23 \mathrm{C}\end{array}$ & Coloniale & $\begin{array}{c}7.5 \text { YR7/6 } \\
\text { Reddish Yellow }\end{array}$ & $/$ & $375-350$ & $\begin{array}{c}\text { Howland 1958, 60, pls. 8, 36, } \\
\text { n. 229 }\end{array}$ & 1 \\
\hline 49 & $\begin{array}{c}\text { Lucerna tipo } \\
23 \mathrm{D}\end{array}$ & Coloniale & $\begin{array}{c}7.5 \text { YR7/6 } \\
\text { Reddish Yellow }\end{array}$ & $/$ & $350-325$ & $\begin{array}{c}\text { Howland 1958, 61-62, pls. 8, } \\
37, \text { n. 238 }\end{array}$ & 1 \\
\hline 50 & Lopadion & Coloniale & $\begin{array}{c}5 \text { YR8/4 } \\
\text { Pink }\end{array}$ & 12 & $400-375$ & $\begin{array}{c}\text { Mozia XIII, Tav. XXXII, } \\
\text { MF.03.1219/10 }\end{array}$ & 1 \\
\hline 51 & Lekanis & Coloniale & $\begin{array}{c}7.5 Y R 7 / 4 \\
\text { Pink }\end{array}$ & 10 & $400-375$ & $\begin{array}{c}\text { Morel 1981, 327, pl. 143, } \\
4713 a 1\end{array}$ & 1 \\
\hline
\end{tabular}

